\title{
Quantum Monodromy in Prolate Ellipsoidal Billiards
}

\author{
Holger Waalkens \\ Institut für Theoretische Physik and Institut für Dynamische Systeme, Universität Bremen, Bremen, Germany \\ E-mail: waalkens@physik.uni-bremen.de
}

and

Holger R. Dullin

Department of Mathematical Sciences, Loughborough University, Leicestershire, United Kingdom

Received May 16, 2001

\begin{abstract}
This is the third in a series of three papers on quantum billiards with elliptic and ellipsoidal boundaries. In the present paper we show that the integrable billiard inside a prolate ellipsoid has an isolated singular point in its bifurcation diagram and, therefore, exhibits classical and quantum monodromy. We derive the monodromy matrix from the requirement of smoothness for the action variables for zero angular momentum. The smoothing procedure is illustrated in terms of energy surfaces in action space including the corresponding smooth frequency map. The spectrum of the quantum billiard is computed numerically and the expected change in the basis of the lattice of quantum states is found. The monodromy is already present in the corresponding two-dimensional billiard map. However, the full three degrees of freedom billiard is considered as the system of greater relevance to physics. Therefore, the monodromy is discussed as a truly three-dimensional effect. () 2002 Elsevier Science (USA)
\end{abstract}

\section{INTRODUCTION}

Even though quantum monodromy is present in the quantization of many old and prominent examples of classical mechanics, most notably the spherical pendulum, it had not been found until 1988 by Cushman and Duistermaat [1]. The origin of the phenomenon is that the action-angle variables of a completely integrable system need not be globally defined even in the absence of singularities of the energy momentum mapping. This question was first studied by Duistermaat [2]. The reason that monodromy was not observed in the classical works might be that in the tradition of analytical mechanics action variables were more a device for the integration of the system than an object with interesting global geometric properties.

The geometrisation of mechanics started with Arnold [3]. The Liouville-Arnold theorem shows that in a neighborhood of a regular point of the energy momentum mapping action-angle variables exist. However, they are only unique up to unimodular transformations and there is no statement about their global properties (or even existence). The study of global properties of integrable mechanical systems has been developed in (among others) [4,5]. The energy momentum mapping of a completely integrable system is the map from phase space to the constants of motion. A regular value of this map has as preimage a collection of $d$-tori where $d$ is the number of degrees of freedom. The image of the map is a subset of $\mathbb{R}^{d}$. For $d=2$ an equilibrium point with a complex quadruplet of eigenvalues is an example of a critical point in phase space that has an isolated critical value in the image. This 
gives the possibility to encircle the isolated point in the image. The circle is started with a certain choice of fundamental cycles on the torus (and hence corresponding actions). Smoothly deforming this basis of cycles around the critical value monodromy occurs if the basis has changed when returning to the initial torus. The unimodular matrix that transforms the old into the new cycles is called the monodromy matrix.

Classical monodromy has been reported for many systems (see [5, 6] and the references therein) and for two degrees of freedom the monodromy theorem [7] states that if the preimage of the isolated critical point is a torus with $n$ pinches then the monodromy matrix is $\left(\begin{array}{ll}1 & 0 \\ n & 1\end{array}\right)$. A good method to calculate the number of pinches is via singular reduction; see [5] for the details.

Quantum monodromy is a related change in the basis of the lattice of eigenvalues of the corresponding commuting operators. The obvious connection between classical and quantum monodromy is via semiclassical EBK quantization of the actions as it was done in [1,8]. A rigorous treatment using microlocal analysis instead is given in [9].

In this paper we are going to show that the quantum problem of a particle confined to a prolate ellipsoid in $\mathbb{R}^{3}$ exhibits monodromy. The corresponding classical problem is the billiard inside that region. This system has three degrees of freedom but due to the hard walls of the ellipsoid it is not a smooth system. This is the reason why we resort to the analysis of actions to calculate monodromy. Let us remark that this is the third paper in a series that deals with ellipsoidal quantum billiards $[10,11]$. Our method rests on separability of the system. From separation we define what we call natural actions. Besides the critical values of the energy momentum mapping these actions can have additional singularities related to singularities in the separating coordinate system. Namely, they are continuous but not differentiable for angular momentum zero. This phenomenon has been first observed in [12] for the Lagrange top, but was at that time not related to monodromy. In fact, this is a more general phenomenon that also occurs in the Kovalevskaya top [13,14] where (within the current framework of the theory) there is no monodromy.

From the analytical point of view the additional singularities of the natural actions occur when in an action integral of third kind a branch point collides with a pole. The isolated point in the bifurcation diagram occurs when two branch points collide with a pole. This seems to be a general mechanism leading to monodromy.

Ellipsoidal quantum billiards and, in particular, quantum billiards with a prolate ellipsoidal boundary serve as model systems in various areas of physics. We mention the jellium model of metal clusters $[15,16]$, the similarity between shell structures of prolate ellipsoidal cavities and the Woods-Saxon potential of nuclear physics [17, 18], and the quasi particle dynamics in Bose condensates [19]. The current paper may contribute to the classification of the quantum states in these systems.

The outline of this paper is as follows. In Section 2 the prolate ellipsoidal billiard is introduced as a classical system whose equations of motion are separated in elliptic coordinates. Its bifurcation diagram is derived from the corresponding billiard map. In Section 3 the classical monodromy of the system is calculated from the requirement of the existence of locally smooth actions. In Section 4 the monodromy is then further discussed in terms of energy surfaces in action space and the corresponding frequency map. In particular, the examination of three invariant two degrees of freedom subsystems included in the three degrees of freedom billiard flow gives a kind of dynamical description of the monodromy. In Section 5 the quantum mechanical version of the billiard is introduced and the implication of monodromy on the quantum spectrum is discussed. Concluding remarks and an outlook are given in Section 6.

\section{THE CLASSICAL SYSTEM}

The prolate ellipsoidal billiard is defined as follows. Consider a prolate ellipsoid in $\mathbb{R}^{3}$ with coordinates $\boldsymbol{r}=(x, y, z)^{t}$ defined by 


$$
\mathcal{E}_{1}(\boldsymbol{r})=x^{2}+\frac{y^{2}+z^{2}}{1-a^{2}}-1=0 \quad\left(a^{2}<1\right) .
$$

A particle of mass 1 with momentum $\boldsymbol{p}=\left(p_{x}, p_{y}, p_{z}\right)^{t}$ moves on straight lines in the direction of $\boldsymbol{p}$ with velocity $|\boldsymbol{p}|$ inside the ellipsoid. Encountering the boundary at $\boldsymbol{r}$ the momentum is reflected according to the reflection map

$$
R(\boldsymbol{r}, \boldsymbol{p})=\boldsymbol{p}-2(\boldsymbol{p}, \boldsymbol{n}(\boldsymbol{r})) \boldsymbol{n}(\boldsymbol{r}), \quad \boldsymbol{n}(\boldsymbol{r})=c\left(\boldsymbol{r}-a^{2} x \boldsymbol{e}_{x}\right),
$$

where $\boldsymbol{n}(\boldsymbol{r})$ is the normal at the reflection point, $c^{2}=1 /\left(1-a^{2}\right)$ is a normalization constant, and $(\cdot, \cdot)$ is the standard Euclidean scalar product. The billiard can be considered as a singular limit of the geodesic flow on an ellipsoid in $\mathbb{R}^{4}$ with two equal middle axes where the shortest semi-axis collapses to zero.

\subsection{Billiard Map}

For the derivation of the bifurcation diagram below it is useful to forget about the trivial free flight inside the billiard and to consider instead the discrete system from reflection to reflection. This gives the billiard map $P$ which is defined by moving from a point $\boldsymbol{r}$ on the boundary $\mathcal{E}_{1}(\boldsymbol{r})=0$ in an inward direction $\boldsymbol{p}((\boldsymbol{p}, \boldsymbol{n}(\boldsymbol{r})) \leq 0)$ until the moment right after the next reflection. The energy $E=\boldsymbol{p}^{2} / 2$ which determines the velocity is a constant of no interest for the billiard map. For definiteness we choose $E=1 / 2$. Then $P$ acts on the phase space

$$
\left\{(\boldsymbol{r}, \boldsymbol{p}): E=1 / 2, \mathcal{E}_{1}=0,(\boldsymbol{p}, \boldsymbol{n}(\boldsymbol{r})) \leq 0\right\}
$$

This phase space has the topology of $T^{*} S^{2}$ where from the cotangent space at each point on the sphere $S^{2}$ only the closed disk of radius 1 is allowed. Hence, it is a closed compact manifold with boundary, the unit disk bundle of $S^{2}$. The center of the disk corresponds to a motion perpendicular to the billiard boundary. The phase space boundary contains all the motions that are tangent to the billiard boundary.

The billiard map $P$ is completely integrable. With the angular momenta

$$
L=r \times p, \quad L_{+}=(r-a) \times p, \quad L_{-}=(r+a) \times p
$$

about the origin and the two focus points of the prolate ellipsoid at $\pm \boldsymbol{a}=( \pm a, 0,0)^{t}$ the constants of motion are $L_{x}=y p_{z}-z p_{y}$ and

$$
K=\left(\boldsymbol{L}_{-}, \boldsymbol{L}_{+}\right)+2 a^{2} E=\boldsymbol{L}^{2}+a^{2} p_{x}^{2} .
$$

In the limit of a spherical billiard, $a \rightarrow 0, K$ becomes the square of the total angular momentum. Both $K$ and $L_{z}$ are trivially constant along the free flight $\boldsymbol{r}+t \boldsymbol{p}$. The non-trivial property is the invariance under the reflection map $\boldsymbol{p}^{\prime}=R(\boldsymbol{r}, \boldsymbol{p})$. The invariance of $L_{x}$ immediately follows from $(\boldsymbol{r} \times \boldsymbol{n}(\boldsymbol{r}))_{x}=0$. For $K$ we have to check that $K=K^{\prime}$, i.e.,

$$
\left(\boldsymbol{L}_{-}, \boldsymbol{L}_{+}\right)=\left(\boldsymbol{L}_{-}+\Delta(a x+1), \boldsymbol{L}_{+}+\Delta(a x-1)\right), \quad \Delta=2 c(\boldsymbol{p}, \boldsymbol{n}) \boldsymbol{r} \times \boldsymbol{a} .
$$

After some simplification this is equivalent to

$$
4 a^{2} c x(\boldsymbol{p}, \boldsymbol{n}) \mathcal{E}_{1}(\boldsymbol{r})\left(\left(y^{2}+z^{2}\right) p_{x}+x\left(a^{2}-1\right)\left(y p_{y}+z p_{z}\right)\right)=0,
$$


so that $K$ is a constant of motion on the ellipsoid $\mathcal{E}_{1}(\boldsymbol{r})=0 . K$ and $L_{x}$ commute with respect to the standard Poisson bracket on $\mathbb{R}^{3}$ because $L_{x}$ commutes with $\boldsymbol{L}^{2}$ and also with $p_{x}$.

\subsection{Separation}

The billiard system can be separated in prolate ellipsoidal coordinates; see, e.g., [20]. The coordinate surfaces of prolate ellipsoidal coordinates are given as a family of confocal rotationally symmetric quadrics

$$
\mathcal{E}_{s}(\boldsymbol{r})=\frac{x^{2}}{s^{2}}+\frac{y^{2}+z^{2}}{s^{2}-a^{2}}-1=0,
$$

with family parameter $s \in\{\xi, \eta\}$. For $a^{2} \leq \xi^{2} \leq 1$ the surfaces $\mathcal{E}_{\xi}(\boldsymbol{r})=0$ are confocal prolate ellipsoids; see Fig. 1a. Their intersections with the $(x, y)$-plane and $(x, z)$-plane are planar ellipses with foci $(x, y)=( \pm a, 0)$ and $(x, z)=( \pm a, 0)$, respectively. Their intersections with the $(y, z)$-plane are circles of radius $\sqrt{\xi^{2}-a^{2}}$. $\xi^{2}=1$ gives the billiard boundary $\mathcal{E}_{1}(\boldsymbol{r})=0$. For $0 \leq \eta^{2} \leq a^{2}$ the surfaces $\mathcal{E}_{\eta}(\boldsymbol{r})=0$ are rotationally symmetric two sheeted hyperboloids; see Fig. 1b. Their intersections with the $(x, y)$-plane and $(x, z)$-plane are confocal hyperbolas with foci at $(x, y)=$ $( \pm a, 0)$ and $(x, z)=( \pm a, 0)$, respectively.

With $\varphi$ giving the angle about the $x$-axis the prolate ellipsoidal coordinates $\boldsymbol{q}=(\varphi, \eta, \xi)^{t}$ are related to the Euclidean coordinates by the transformation

$$
(x, y, z)=\frac{1}{a}\left(\xi \eta, \sqrt{\left(\xi^{2}-a^{2}\right)\left(a^{2}-\eta^{2}\right)} \cos \varphi, \sqrt{\left(\xi^{2}-a^{2}\right)\left(a^{2}-\eta^{2}\right)} \sin \varphi\right)
$$

whereby the two sheets of the $\eta$-hyperboloids are distinguished by different signs of $\eta$. The coordinate ranges

$$
-a \leq \eta \leq a \leq \xi \leq 1, \quad 0 \leq \varphi \leq 2 \pi
$$

yield a full cover of the interior of the prolate ellipsoid. The coordinate transform (9) is singular for $\eta= \pm a$ and for $\xi=a$, i.e., along the $x$-axis.

Extending the coordinate transformation (9) to a canonical point transformation gives the old momenta $\boldsymbol{p}=(\partial \boldsymbol{r} / \partial \boldsymbol{q})^{-t} \boldsymbol{p}_{q}$ in terms of the new momenta $\boldsymbol{p}_{q}=\left(p_{\varphi}, p_{\eta}, p_{\xi}\right)^{t}$. The new Hamiltonian

a)

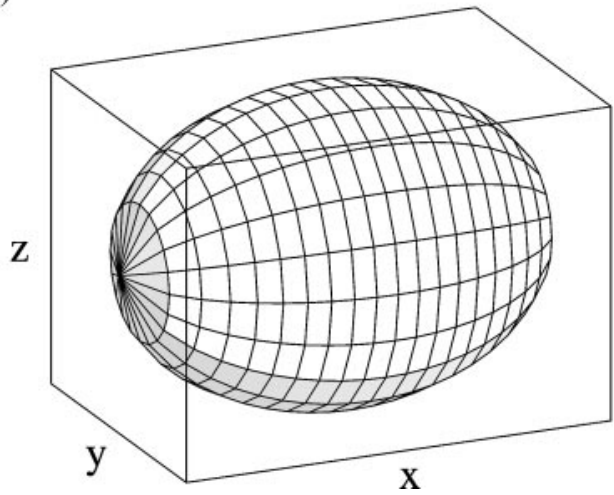

b)

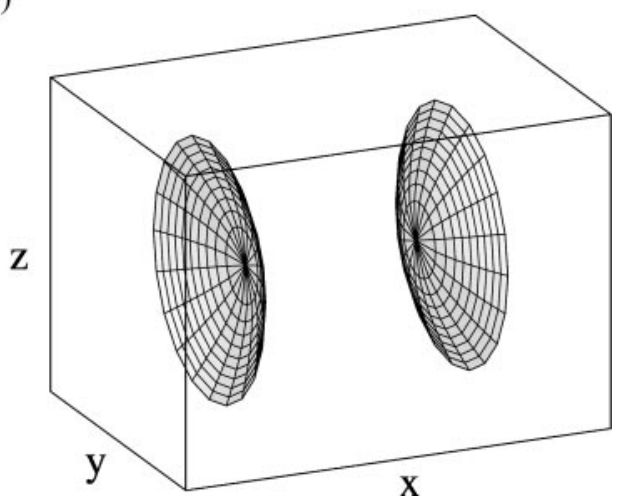

FIG. 1. Surfaces of the prolate ellipsoidal coordinates: $\xi$-ellipsoid (a) and two-sheeted $\eta$-hyperboloid (b). 
describing the free motion becomes

$$
H=\frac{1}{2} \frac{1}{\xi^{2}-\eta^{2}}\left(\left(\xi^{2}-a^{2}\right) p_{\xi}^{2}+\left(a^{2}-\eta^{2}\right) p_{\eta}^{2}\right)+\frac{a^{2}}{2} \frac{p_{\varphi}^{2}}{\left(\xi^{2}-a^{2}\right)\left(a^{2}-\eta^{2}\right)}
$$

The free motion generated by this Hamiltonian can easily be separated. In addition, the momentum change upon a reflection at the boundary $\xi=1$ is simply given by the sign change

$$
\left(p_{\varphi}, p_{\eta}, p_{\xi}\right) \mapsto\left(p_{\varphi}, p_{\eta},-p_{\xi}\right)
$$

Therefore these coordinates separate both the free motion and the reflection condition.

Below we find it useful to consider another parametrization of the separating coordinates $(\eta, \xi)$ given by

$$
(\eta, \xi)=(a \sin \psi, a \cosh \rho)
$$

with conjugate momentum variables $\left(p_{\psi}, p_{\rho}\right)=\left(\sqrt{a^{2}-\eta^{2}} p_{\eta}, \sqrt{a^{2}-\xi^{2}} p_{\xi}\right)$ (see, e.g., [10]).

Since both the Hamiltonian $H$ and the reflection condition (12) are independent of $\varphi$ the angle $\varphi$ is cyclic and the momentum component $p_{\varphi}$, which is the angular momentum $L_{x}$ about the $x$-axis, is a constant of motion. Multiplying Eq. (11) by $\xi^{2}-\eta^{2}$ we obtain the separation constant

$$
\begin{aligned}
K & =2 E \xi^{2}-\left(\xi^{2}-a^{2}\right) p_{\xi}^{2}-a^{2} \frac{p_{\varphi}^{2}}{\xi^{2}-a^{2}} \\
& =2 E \eta^{2}+\left(a^{2}-\eta^{2}\right) p_{\eta}^{2}+a^{2} \frac{p_{\varphi}^{2}}{a^{2}-\eta^{2}}
\end{aligned}
$$

which is the $K$ previously defined in (5).

\subsection{Bifurcation Diagram}

The energy momentum mapping $\mathcal{M}$ of the discrete integrable system is the map from phase space (3) to the values of the constants of motion $L_{x}$ and $K$. Phase space is foliated into invariant manifolds given by the preimages of $\mathcal{M}$. The invariant manifold that is a preimage of a regular value of $\mathcal{M}$ is a collection of 2-tori. This follows from the discrete analogue of the Liouville-Arnold theorem; see, e.g., [21]. Critial points of $\mathcal{M}$ are determined by

$$
\operatorname{rank} \frac{\partial\left(E, \mathcal{E}_{1}, L_{x}, K\right)}{\partial(\boldsymbol{r}, \boldsymbol{p})}<4
$$

with $(\boldsymbol{r}, \boldsymbol{p})$ from phase space (3). For the calculation of critical points it is useful to stick to the separating coordinates whenever they are non-singular and else turn to Euclidean variables $(x, y, z)$.

In the separating coordinates the condition $\mathcal{E}_{1}=0$ is satisfied by restricting to $\xi=1$. The condition $E=1 / 2$ is used to eliminate $p_{\xi}$ in $K$; i.e., we use (15). This elimination is singular when $p_{\xi}=0$, i.e., for motions tangent to the billiard boundary. Note that $\xi=1, p_{\xi}=0$ describe the boundary of phase space. Using $\psi$ of Eq. (13) instead of $\eta$ in (15) gives

$$
K=2 E a^{2} \sin ^{2} \psi+a^{2} p_{\psi}^{2}+\frac{p_{\varphi}^{2}}{\cos ^{2} \psi}
$$


Critical points occur when $p_{\psi}=0$ and $\psi=0 \bmod \pi / 2$, i.e., at $\eta=0, \pm a$. For $\eta=0$ this gives $K=p_{\varphi}^{2}$ which describes motion in the equatorial plane $x=0$. In the other canonical plane $\left(\varphi, p_{\varphi}\right)$ we never have critical points because $p_{\varphi}$ is a constant of motion. It remains to express the condition $p_{\xi}=0$ in terms of the constants of motion. For this we eliminate $p_{\eta}$ using $E=1 / 2$; i.e., we use (14). With $\xi=1$ and $p_{\xi}=0$ we find $K=2 E-p_{\varphi}^{2} a^{2} /\left(1-a^{2}\right)$.

If there are further critical points they have to lie in the coordinate singularity, i.e., at the intersection of the $x$-axis with the boundary ellipsoid. Condition (16) is equivalent to the existence of a solution with not all $\lambda_{i}$ equal to zero for

$$
\begin{aligned}
\lambda_{2}\left(\boldsymbol{r}-a^{2} x \boldsymbol{e}_{x}\right)+\lambda_{3} \boldsymbol{p} \times \boldsymbol{e}_{x}+\lambda_{4} \boldsymbol{p} \times \boldsymbol{r} \times \boldsymbol{p} & =0 \\
\lambda_{1} \boldsymbol{p}+\lambda_{3} \boldsymbol{e}_{x} \times \boldsymbol{r}+\lambda_{4}\left(\boldsymbol{r} \times \boldsymbol{p} \times \boldsymbol{r}+a^{2} \boldsymbol{e}_{x} p_{x}\right) & =0 .
\end{aligned}
$$

For $(x, y, z)=( \pm 1,0,0)$ one finds by direct calculation that the only solution besides the tangent case has $p_{y}=p_{z}=0$ for which the gradients of $L_{x}$ and $K$ vanish identically. There is no other solution.

Therefore, there are only three critical cases

$$
\begin{aligned}
& I:\left(\boldsymbol{p}, \boldsymbol{r}-a^{2} x \boldsymbol{e}_{x}\right)=0, \\
& I I: x=p_{x}=0, \\
& I I I: y=z=p_{y}=p_{z}=0 .
\end{aligned}
$$

The constants of motion $K$ and $L_{x}=p_{\varphi}$ are homogeneous functions of the momenta. It is convenient to scale the momenta by energy and introduce

$$
\left(l_{\varphi}, \kappa^{2}\right)=\left(\frac{L_{x}}{\sqrt{2 E}}, \frac{K}{2 E}\right)
$$

as new constants of motion. The square in Eq. (23) indicates that $K$ is a positive function on phase space with dimension of a squared angular momentum. In terms of the constants $\left(l_{\varphi}, \kappa^{2}\right)$ the images of the critical points $I, I I$, and $I I I$ are

$$
\begin{aligned}
& I: \kappa^{2}=1-\frac{a^{2} l_{\varphi}^{2}}{1-a^{2}}, \\
& I I: \kappa^{2}=l_{\varphi}^{2}, \\
& \text { III: }\left(l_{\varphi}, \kappa^{2}\right)=\left(0, a^{2}\right) .
\end{aligned}
$$

The set of critical values $I \cup I I \cup I I I$ is called the bifurcation diagram which we show in Fig. 2 . The parabolas $\overline{A B A^{\prime}}$ and $\overline{A C A^{\prime}}$ correspond to cases $I$ and $I I$, respectively. The isolated point $F$ (a so-called focus point) represents case III. Each interior regular value in Fig. 2 represents an invariant 2 -torus of the billiard map. Because of the isolated critical value $F$ the interior region is not simply connected; i.e., cycles encircling $F$ cannot be contracted to a point. This is the major condition for the appearance of monodromy as will be explained in Sections 3 and 5.

Case $I$, the upper parabola $\overline{A B A^{\prime}}$, corresponds to the geodesic flow on the boundary of the billiard. From the point of view of the billiard map this is singular because the time between reflections becomes zero so that every point becomes a fixed point. Note that phase space is a compact manifold (the unit disk bundle of $S^{2}$ ) whose boundary is the unit cotangent bundle of $S^{2}$, and case $I$ corresponds to the boundary, which is invariant under the dynamics. The continuous dynamics on this invariant set is the geodesic flow on the billiard boundary. 


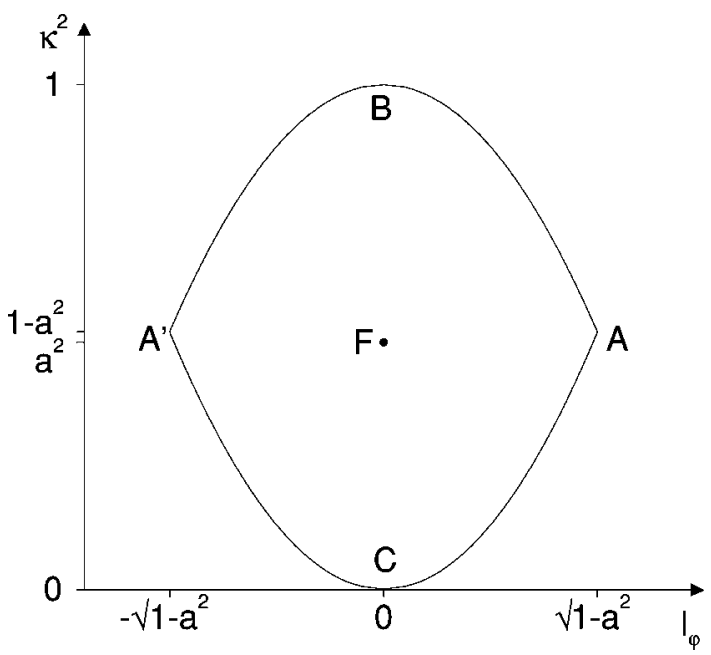

FIG. 2. Bifurcation diagram of the billiard map in the $\left(l_{\varphi}, \kappa^{2}\right)$-plane.

Case $I I$, the lower parabola $\overline{A C A^{\prime}}$, is the motion in the equatorial plane $x=0$, which is bounded by a circle of radius $\sqrt{1-a^{2}}$. The billiard map restricted to the invariant set $I I$ is just the billiard map of the circle. The invariant sets in the preimage of $I I$ are 1-tori. Cases $I$ and $I I$ intersect at the points $\left(l_{\varphi}, \kappa^{2}\right)=\left( \pm \sqrt{1-a^{2}}, 1-a^{2}\right)$ which represent a line of fixed points in the plane $x=0$ at the boundary of phase space.

Case III, the isolated critical value $F$, has as preimage the period two points with $x= \pm 1$ and its stable and unstable manifolds. The structure of the preimage of $F$ can be understood as follows. Because of the rotational symmetry of the ellipsoid we can reduce by the $S^{1}$ symmetry and obtain a family of billiards in the ellipse with an additional effective potential proportional to $l_{\varphi}^{2}$. Each reduced map is described by a Poincaré map from $S^{1} \times D^{1}$ to itself. The corresponding leaf $l_{\varphi}=$ const in the full phase space of the billiard map $\mathcal{P}$ is obtained from this by letting the flow generated by $L_{x}$ act on a representative of the reduced space in full phase space. Because the symmetry axis is fixed under this flow this effectively results in a rotation of the reduced Poincaré map. In particular for $l_{\varphi}=0$ we obtain the billiard map of the ordinary billiard in the ellipse as a reduced map. Its phase space is foliated by two types of 1-tori which are the level sets of $K$ shown in Fig. 3a. The separatrix with $K=2 a^{2} E$ is given by $p_{\psi}^{2} /(2 E)=\cos ^{2} \psi$, and the crossings of these curves mark the period two points of the critical orbit. Letting $L_{x}$ act on this reduced phase space gives a solid 2-torus embedded in the full phase space (3) and inside this solid torus is a doubly pinched 2-torus,

a)

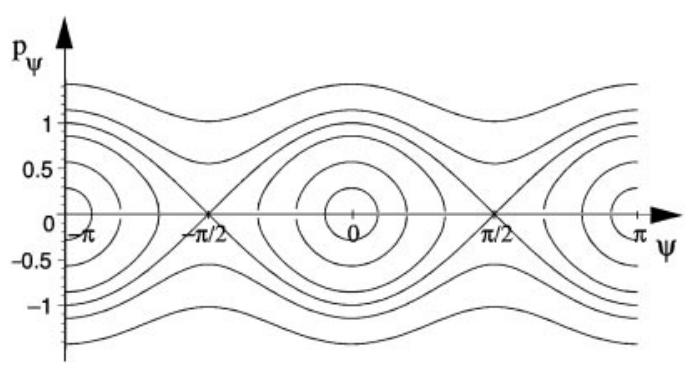

b)

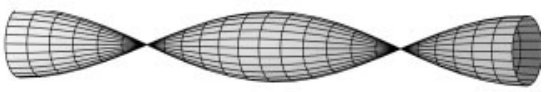

c)

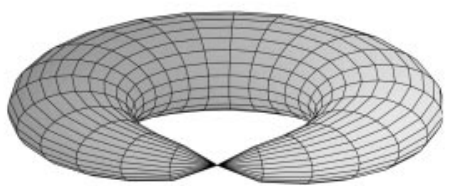

FIG. 3. (a) Invariant 1-tori of the billiard map for $l_{\varphi}=0$, (b) the doubly pinched 2-torus derived from rotating the separatrix in (a), and (c) the corresponding singly pinched 2-torus from incorporating the action of the billiard map. 
the two pinches corresponding to the two period 2 points on the symmetry axis; see Fig. $3 b$. The invariant 2-torus with only one pinch shown in Fig. 3c is obtained if we factor by the action of the map; i.e., we identify points on the doubly pinched torus that are mapped into each other.

From the general monodromy theorem [7] we expect to find a monodromy of 1, because we have a torus with a single pinch as the preimage of the isolated critical value. However, this theorem does not apply in our setting, because we are dealing with a map and with a phase space with boundary. In the next subsection we come back to the full billiard flow for which we will derive the monodromy in the following section directly from its actions.

\subsection{The Billiard Flow}

Because $\kappa^{2}$ and $l_{\varphi}$ both scale with $E$ the bifurcation diagram for the three degrees of freedom system is like a cylinder with cross section shown in Fig. 2 with a bottom $E=0$ where there is no motion at all. The focus point $F$ then becomes a line of focus points.

The caustics of this three degrees of freedom system can be discussed in terms of the roots of the polynomial

$$
f\left(s^{2}\right)=s^{4}-\left(\kappa^{2}+a^{2}\right) s^{2}+a^{2}\left(\kappa^{2}-l_{\varphi}^{2}\right) .
$$

By Eqs. (14) and (15) it allows us to give the separated momenta $p_{\eta}$ and $p_{\xi}$ the compact form

$$
\frac{p_{s}^{2}}{2 E}=\frac{f\left(s^{2}\right)}{\left(s^{2}-a^{2}\right)^{2}}=\frac{\left(s^{2}-s_{1}^{2}\right)\left(s^{2}-s_{2}^{2}\right)}{\left(s^{2}-a^{2}\right)^{2}},
$$

where $s \in\{\eta, \xi\}$ and

$$
s_{1,2}^{2}=\frac{1}{2}\left(a^{2}+\kappa^{2} \mp \sqrt{\left(a^{2}-\kappa^{2}\right)^{2}+4 a^{2} l_{\varphi}^{2}}\right)
$$

are the roots of $f\left(s^{2}\right)$. For Eq. (28) to give real valued momenta for some configuration $-a \leq \eta \leq$ $a \leq \xi \leq 1$ the roots have to satisfy

$$
0 \leq s_{1}^{2} \leq a^{2} \leq s_{2}^{2} \leq 1
$$

see Fig. 4. Comparing coefficients of $s^{2}$ in (28) gives

$$
\begin{aligned}
l_{\varphi}^{2} & =s_{1}^{2}+s_{2}^{2}-a^{2}-\frac{s_{1}^{2} s_{2}^{2}}{a^{2}}, \\
\kappa^{2} & =s_{1}^{2}+s_{2}^{2}-a^{2} .
\end{aligned}
$$

The bifurcation diagram in Fig. 2 essentially maps to the boundaries of Eq. (30); see Fig. 5. This presentation of the bifurcation diagram is useful for numerical purposes. It is, however, not a proper bifurcation diagram because the constants of motion $s_{1}, s_{2}$ are not smooth function on phase space. The additional edges $\overline{F C}$ and $\overline{F B}$ are a consequence of the singularities of the transformation from $\left(s_{1}^{2}, s_{2}^{2}\right)$ to $\left(l_{\varphi}, \kappa^{2}\right)$ along the line $l_{\varphi}=0$ or, equivalently, $s_{1}^{2}=a^{2}$ and $s_{2}^{2}=a^{2}$. Because the diagram in Fig. 5 does not distinguish between different signs of $l_{\varphi}$ each of its interior points represents two 3-tori which differ by their sense of rotation about the $x$-axis. In Fig. 6 we present the envelopes of two such 3-tori. An envelope is bounded by the quadrics $\mathcal{E}_{s_{1}}(\boldsymbol{r})=0, \mathcal{E}_{s_{2}}(\boldsymbol{r})=0$, and $\mathcal{E}_{1}(\boldsymbol{r})=0$, where the former two quadrics are a caustic and the latter is the billiard boundary. For the billiard map the caustic is the annulus defined by $\eta= \pm s_{1}$ on the ellipsoid. 


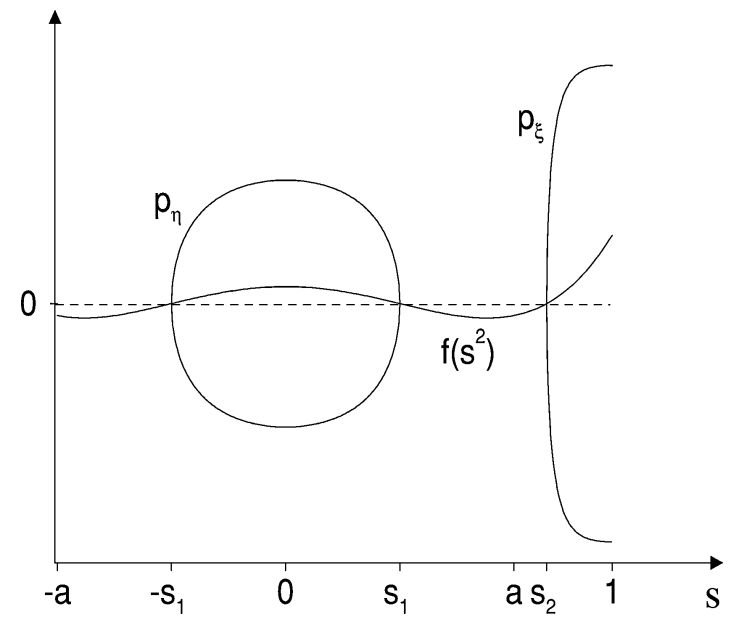

FIG. 4. The polynomial $f\left(s^{2}\right)$ and the phase portraits $\left(\eta, p_{\eta}\right)$ and $\left(\xi, p_{\xi}\right)$.

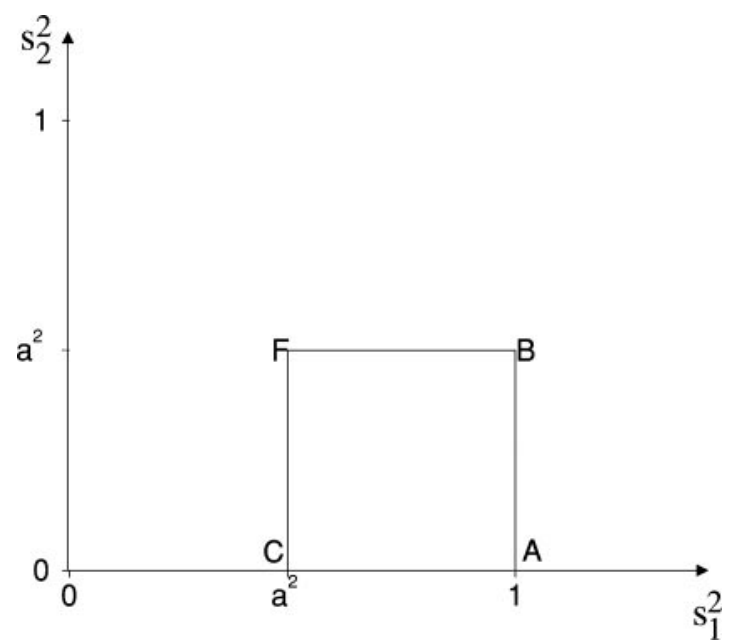

FIG. 5. Phase diagram in the $\left(s_{1}^{2}, s_{2}^{2}\right)$-plane.

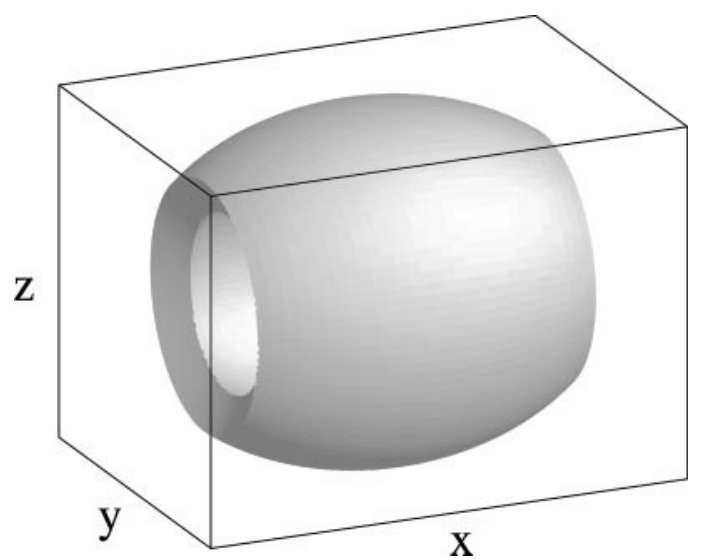

FIG. 6. Envelope in the Euclidean configuration space of a regular 3-torus of the prolate ellipsoidal billiard. 


\section{CLASSICAL MONODROMY}

The Liouville-Arnold theorem [3] states that for regular values of the energy momentum mapping the preimage is a union of tori if it is compact and that there exist action-angle variables in the neighborhood of these tori. For a separable system the explicit construction of actions is straightforward. However, there are two objections: (1) action-angle variables often are not globally singularity free coordinates on phase space, and (2) action-angle variables related to the (often not globally singularity free) separating coordinate system need not be smooth. In the following we will make this more precise and relate it to monodromy.

\subsection{Natural Actions}

Action variables are obtained from integrating the differential $\boldsymbol{p} \mathrm{d} \boldsymbol{q}$ along independent cycles on a torus. The natural actions of a separable system are obtained by using a basis of cycles $C_{i}$ whose projections onto configuration space coincide with the coordinate lines of the separating coordinate system. For the billiard in the prolate ellipsoid we obtain

$$
C_{1}: \mathrm{d} \eta=\mathrm{d} \xi=0, \quad C_{2}: \mathrm{d} \varphi=\mathrm{d} \xi=0, \quad C_{3}: \mathrm{d} \varphi=\mathrm{d} \eta=0 .
$$

From Eq. (11) we see that the energy dependence can be removed by scaling the momentum variables. Taking into account that $\eta$ oscillates between $-s_{1}$ and $+s_{1}$ and $\xi$ oscillates between $s_{2}$ and the reflection at the billiard boundary $\xi=1$ the energy scaled action variables read

$$
\begin{aligned}
& I_{1} \equiv \frac{1}{\sqrt{2 E}} I_{\varphi}=\frac{1}{2 \pi} \oint_{C_{1}} \frac{p_{\varphi}}{\sqrt{2 E}} \mathrm{~d} \varphi=l_{\varphi}, \\
& I_{2} \equiv \frac{1}{\sqrt{2 E}} I_{\eta}=\frac{1}{2 \pi} \oint_{C_{2}} \frac{p_{\eta}}{\sqrt{2 E}} \mathrm{~d} \eta=\frac{1}{\pi} \int_{-s_{1}}^{s_{1}} \frac{\sqrt{f\left(\eta^{2}\right)}}{a^{2}-\eta^{2}} \mathrm{~d} \eta, \\
& I_{3} \equiv \frac{1}{\sqrt{2 E}} I_{\xi}=\frac{1}{2 \pi} \oint_{C_{3}} \frac{p_{\xi}}{\sqrt{2 E}} \mathrm{~d} \xi=\frac{1}{\pi} \int_{s_{2}}^{1} \frac{\sqrt{f\left(\xi^{2}\right)}}{\xi^{2}-a^{2}} \mathrm{~d} \xi .
\end{aligned}
$$

The integrants of $I_{2}$ and $I_{3}$ are identical up to a sign and of elliptic type. The integral $I_{2}$ is complete; i.e., it is integrated between two branch points. $I_{3}$ is incomplete because of the billiard boundary. Due to the poles at $\pm a$ the integrals are of the third kind, which seems to be an essential feature for the appearance of monodromy. For the billiard in the triaxial ellipsoid discussed in [11] the integrals are hyperelliptic but of the second kind. The introduction of rotational symmetry creates a pole in the integrand, whose residue is the angular momentum $l_{\varphi}=I_{1}$ with respect to the symmetry axis. For completeness we also mention the expressions in terms of Legendre standard integrals as given in [22]. One finds

$$
\begin{aligned}
I_{2}= & \frac{2}{\pi s_{2}}\left(\left(s_{1}^{2}-a^{2}+\frac{a^{2} l_{\varphi}^{2}}{s_{2}^{2}-a^{2}}\right) \mathcal{K}(k)+s_{2}^{2} \mathcal{E}(k)-\frac{a^{2} l_{\varphi}^{2}\left(s_{2}^{2}-s_{1}^{2}\right)}{\left(s_{2}^{2}-a^{2}\right)\left(a^{2}-s_{1}^{2}\right)} \Pi\left(-\beta^{2}, k\right)\right), \\
I_{3}= & \frac{1}{\pi s_{2}}\left(\left(a^{2}-s_{1}^{2}+\frac{a^{2} l_{\varphi}^{2}}{a^{2}-s_{1}^{2}}\right) \mathcal{F}(\chi, k)-s_{2}^{2} \mathcal{E}(\chi, k)+s_{2} \sin \chi\right. \\
& \left.-\frac{a^{2} l_{\varphi}^{2}\left(s_{2}^{2}-s_{1}^{2}\right)}{\left(s_{2}^{2}-a^{2}\right)\left(a^{2}-s_{1}^{2}\right)} \Pi\left(\chi,-\gamma^{2}, k\right)\right),
\end{aligned}
$$


where $\mathcal{F}(\chi, k), \mathcal{E}(\chi, k)$, and $\Pi(\chi, n, k)$ are Legendre's incomplete elliptic integrals of the first, second, and third kind, respectively, with modulus $k$, amplitude $\chi$, and parameter $n$ in the notation of [23]. $\mathcal{K}(k), \mathcal{E}(k)$, and $\Pi(n, k)$ are the corresponding complete elliptic integrals. The modulus, the parameters, and the amplitude are

$$
k=\frac{s_{1}}{s_{2}}, \quad \beta^{2}=\frac{s_{2}^{2}-a^{2}}{a^{2}-s_{1}^{2}} k^{2}, \quad \gamma^{2}=\frac{a^{2}-s_{1}^{2}}{s_{2}^{2}-a^{2}}, \quad \sin ^{2} \chi=\frac{1-s_{2}^{2}}{1-s_{1}^{2}},
$$

respectively.

In the following we will see that even if we stay away from the critical values of the energy momentum mapping the natural actions $\left(I_{1}, I_{2}, I_{3}\right)$ are functions of $\left(l_{\varphi}, \kappa^{2}\right)$ with discontinuous derivatives. To be more precise, $I_{2}$ and $I_{3}$ are continuous but not differentiable with respect to $l_{\varphi}$ on the line $l_{\varphi}=0$ in Fig. 2. Except for the three points with $\kappa^{2}=0, a^{2}, 1$ this line does not contain critical values of the energy momentum mapping. This is the objection mentioned above that natural actions of separable systems must not be smooth functions. To see this first note that $I_{2}$ and $I_{3}$ are even in $l_{\varphi}$. For differentiable functions this implies zero derivative at zero. We now show that the derivative from the right is non-zero and hence the actions are not differentiable at $l_{\varphi}=0$. Because of symmetry the derivative from the left just gives the negative of that. The formal derivative is

$$
\frac{\partial I_{i}}{\partial l_{\varphi}}=-\frac{a^{2} l_{\varphi}}{2 \pi} \oint_{C_{i}} \frac{\mathrm{d} s}{\left(a^{2}-s^{2}\right) \sqrt{f\left(s^{2}\right)}},
$$

where there is only one sign for $i=2,3$ if the integration paths $C_{i}$ in the complex plane $\mathbb{C}(s)$ and the branch of $\sqrt{f\left(s^{2}\right)}$ are chosen as in Fig. 7. The rhs of Eq. (40) can be non-zero at $l_{\varphi}=0$ despite the factor $l_{\varphi}$. The reason is that for $l_{\varphi} \rightarrow 0$ the branch points $\pm s_{i}$ approach the poles at $\pm a$. The poles of order $3 / 2$ that develop for the integrand in this limit give a diverging contribution of the integral and the question is how this is compensated by the vanishing prefactor $l_{\varphi}$. To see this it is useful to deform the integration path as to include the pole(s), and then subtract the contribution from loops around the pole(s); see Fig. 7. Then it is generally true that

$$
\oint_{C_{2}}=\oint_{B_{2}}+\oint_{R_{-}}+\oint_{R_{+}}, \quad \oint_{C_{3}}=\oint_{B_{3}}+\oint_{R_{+}},
$$

where the $B_{i}$ enclose the branch cut and the two poles with the same clockwise orientation as $C_{i}$ and the contribution of the two poles at $\pm a$ has to be subtracted by integrating the loops $R_{ \pm}$around them, both in counterclockwise direction.

The contributions from the loops $R_{ \pm}$can be calculated from the residues of the integrand. Because of the square root the integrand is a multivalued function, and we have to be careful to evaluate the residues with a consistent choice of branch. The result is that the square root of the residue at $+a$ has the opposite sign from the one at $-a$. Since $f\left(a^{2}\right)=-a^{2} l_{\varphi}^{2}$ we find

$$
\operatorname{Res}_{s= \pm a} \frac{\mathrm{d} s}{\left(a^{2}-s^{2}\right) \sqrt{f\left(s^{2}\right)}}=\frac{1}{2 i a^{2}\left|l_{\varphi}\right|}
$$

This residue diverges in the limit $l_{\varphi} \rightarrow 0$. However, in Eq. (40) the residue is multiplied by $l_{\varphi}$ so that a finite result is obtained. For the same reason any other contribution to (40) from the regular integrals along the $B_{i}$ vanishes in this limit. 
a)
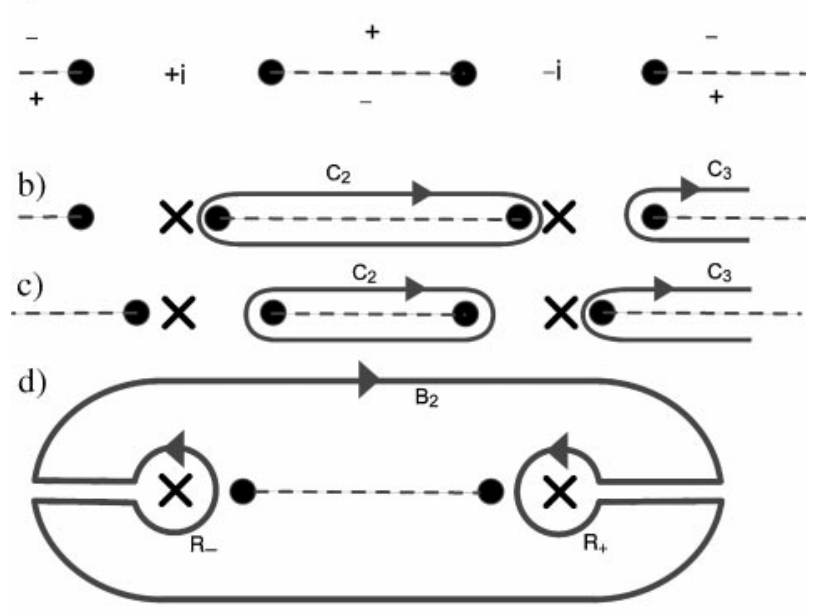

e)

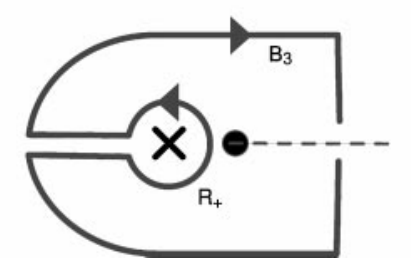

FIG. 7. Complex plane $\mathbb{C}(s)$ and choice of branch of $\sqrt{f\left(s^{2}\right)}$ (a), integration paths for $l_{\varphi}$ near 0 and $\kappa^{2}>a^{2}$ (b), and $\kappa^{2}<a^{2}$ (c). (d) and (e) show the decomposition of $C_{2}$ for case (b) and of $C_{3}$ for case (c), respectively. The dots and the crosses mark the branch points and the poles $-s_{2}<-a<-s_{1}<s_{1}<a<s_{2}$, respectively.

For $\kappa^{2}>a^{2}$ (line $\overline{F B}$ in Fig. 2) the roots $\pm s_{1}$ collide with the poles $\pm a$ from the inside so that for $I_{2}$ we find

$$
\lim _{l_{\varphi} \rightarrow 0^{+}} \frac{\partial I_{2}}{\partial l_{\varphi}}=\lim _{l_{\varphi} \rightarrow 0^{+}}-\frac{a^{2} l_{\varphi}}{2 \pi} 2 \pi i\left(\frac{2}{2 i a^{2}\left|l_{\varphi}\right|}\right)=-1 .
$$

Since $s_{2}^{2}>a^{2}$ the integration path for $I_{3}$ stays away from the pole and therefore $\partial_{l_{\varphi}} I_{3}=0$ on $\overline{F B}$. For $\kappa^{2}<a^{2}$ (line $\overline{F C}$ in Fig. 2) the roots $\pm s_{2}$ collide with the poles $\pm a$ from the outside so that for $I_{3}$ we find

$$
\lim _{l_{\varphi} \rightarrow 0^{+}} \frac{\partial I_{3}}{\partial l_{\varphi}}=\lim _{l_{\varphi} \rightarrow 0^{+}}-\frac{a^{2} l_{\varphi}}{2 \pi} 2 \pi i\left(\frac{1}{2 i a^{2}\left|l_{\varphi}\right|}\right)=-\frac{1}{2} .
$$

Note that the origin of the minus sign in the second formula is the choice of branch. Since the smaller roots satisfy $s_{1}^{2}<a^{2}$, the integration path for $I_{2}$ stays away from the poles and therefore $\partial_{l_{\varphi}} I_{2}=0$ on $\overline{F C}$. The calculations for the limit from the left are the same, so that both functions behave like $c_{i} \operatorname{sgn}\left(l_{\varphi}\right)$, where the constants are $c_{2}=-1$ and $c_{3}=-1 / 2$. A simple way to interpret this result is to say that for $l_{\varphi}>0$ each time the integration path is forced near a pole the integral increases by $-1 / 2$.

Note that the above calculation becomes invalid at the line of focus points $F$. There $\kappa^{2}=a^{2}$, and therefore the branch points $\pm s_{i}$ all collide with the poles $\pm a$. This multiple collision creates a pole of effective order 2 that does produce a singularity in the derivative of the actions. This is an 
indication of the fact that the line of focus points is a line of critical values of the energy momentum mapping.

\subsection{Calculating Monodromy by Smoothing}

The Liouville-Arnold theorem does hold everywhere on the line $l_{\varphi}=0$ except at $\kappa^{2}=0, a^{2}, 1$. Away from these points it guarantees the existence of smooth action variables. The origin for the non-smoothness in the natural actions is that the basis of cycles does not vary smoothly across the coordinate singularity $l_{\varphi}=0$ of the separating coordinate system. A separating coordinate system does distinguish between configuration space and momentum space. In particular the cycles are only defined in their projection to configuration space. For $l_{\varphi}=0$ the complete $\varphi$-cycle projects to a point in configuration space. This is the origin of the non-smoothness of the natural actions.

A smooth basis of cycles in phase space as guaranteed to exist by the Liouville-Arnold theorem can be found by an appropriate unimodular transformation of the cycles of the natural actions. Denote the natural actions for positive $l_{\varphi}$ by $\boldsymbol{I}_{+}$, and those for negative $l_{\varphi}$ by $\boldsymbol{I}_{-}$. Since both $I_{2}$ and $I_{3}$ are independent of the sign of $l_{\varphi}$ and the first action is $l_{\varphi}$ itself we have $\boldsymbol{I}_{-}\left(-l_{\varphi}, \kappa^{2}\right)=\boldsymbol{S} \boldsymbol{I}_{+}\left(l_{\varphi}, \kappa^{2}\right)$ with $S=\operatorname{diag}(-1,1,1)$. We have already seen that on the line $l_{\varphi}=0$ there are different cases depending on whether $\kappa^{2}$ is less or greater than $a^{2}$. In each case we seek a unimodular transformation $M_{i}$ so that $\boldsymbol{I}_{+}$and $\boldsymbol{M}_{i} \boldsymbol{I}_{-}$join smoothly for $I_{1}=l_{\varphi}=0$ :

$$
\begin{array}{ll}
\boldsymbol{I}_{+}=\boldsymbol{M}_{1} \boldsymbol{I}_{-}=\boldsymbol{M}_{1} \boldsymbol{S} \boldsymbol{I}_{+}=\boldsymbol{M}_{1} \boldsymbol{I}_{+} & \text {for } I_{1}=0, \quad \kappa^{2}<a^{2}(\text { line } \overline{F C}), \\
\boldsymbol{I}_{+}=\boldsymbol{M}_{2} \boldsymbol{I}_{-}=\boldsymbol{M}_{2} \boldsymbol{S} \boldsymbol{I}_{+}=\boldsymbol{M}_{2} \boldsymbol{I}_{+} & \text {for } I_{1}=0, \quad \kappa^{2}>a^{2}(\text { line } \overline{F B}) .
\end{array}
$$

Therefore $\left(0, I_{2}, I_{3}\right)^{t}$ must be an eigenvector of $\boldsymbol{M}_{i}$ with eigenvalue 1 . This must be true for all $I_{2}$, $I_{3}$ along the regular part of the line $l_{\varphi}=0$. Both are non-constant and independent functions of $\kappa^{2}$, so that $\left(0, I_{2}, I_{3}\right)^{t}$ must be an eigenvector for arbitrary values of $I_{2}$ and $I_{3}$. This relation and unimodularity of $\boldsymbol{M}_{i}$ requires

$$
\boldsymbol{M}_{i}=\left(\begin{array}{ccc}
1 & 0 & 0 \\
\alpha_{i} & 1 & 0 \\
\beta_{i} & 0 & 1
\end{array}\right)
$$

Now we make use of the fact that $\boldsymbol{I}_{+}$and $\boldsymbol{M}_{i} \boldsymbol{I}_{-}$do not only join continuously but differentiably at $l_{\varphi}=0$, so that

$$
\boldsymbol{M}_{i} \frac{\partial \boldsymbol{I}_{-}}{\partial l_{\varphi}}=\frac{\partial \boldsymbol{I}_{+}}{\partial l_{\varphi}} .
$$

For $\kappa^{2}<a^{2}$ (line $\overline{F C}$ ) we have $\partial_{l_{\varphi}} I_{2}=0$ and from the previous subsection we know $\partial I_{3} / \partial l_{\varphi}=$ $-\operatorname{sgn}\left(l_{\varphi}\right) / 2$. Hence, in $\partial \boldsymbol{I}_{+} / \partial l_{\varphi}$ we have $\partial_{l_{\varphi}} I_{3}=-1 / 2$, while in $\partial_{l_{\varphi}} \boldsymbol{I}_{-}$the sign is changed, $\partial_{l_{\varphi}} I_{3}=$ $1 / 2$. Therefore

$$
\boldsymbol{M}_{1}(1,0,1 / 2)^{t}=(1,0,-1 / 2)^{t} \Rightarrow \alpha_{1}=0, \quad \beta_{1}=-1 .
$$

Similarly, we find for $\kappa^{2}>a^{2}$ (line $\overline{F B}$ )

$$
\boldsymbol{M}_{2}(1,1,0)^{t}=(1,-1,0)^{t} \Rightarrow \alpha_{2}=-2, \quad \beta_{2}=0 .
$$

The monodromy matrix for a counterclockwise cycle around the focus point is obtained from the transformation from $\boldsymbol{I}_{+}$to its smooth continuation across $\overline{F B}$ by $\boldsymbol{M}_{2} \boldsymbol{S}$ followed by the inverse 
transformation $\left(\boldsymbol{M}_{1} \boldsymbol{S}\right)^{-1}$ backwards across $\overline{F C}$, which gives

$$
\boldsymbol{M}=\left(\boldsymbol{M}_{1} \boldsymbol{S}\right)^{-1} \boldsymbol{M}_{2} \boldsymbol{S}=\left(\begin{array}{rrr}
1 & 0 & 0 \\
2 & 1 & 0 \\
-1 & 0 & 1
\end{array}\right)
$$

The monodromy matrix $\boldsymbol{M}$ has eigenvalue one with multiplicity three. We are interested in the normal form of this matrix with respect to conjugation by matrices $\boldsymbol{T} \in S L(3, \mathbb{Z})$. This is like a Jordan normal form where, however, the off-diagonal element cannot be normalized due to the restriction to unimodular transformations. Let us assume a monodromy matrix to be given in the general form where the first column is equal to $(1, \alpha, \beta)^{t}$. If one of $\alpha, \beta$ is zero the matrix is in normal form up to a permutation. Otherwise we have

$$
\boldsymbol{T} \boldsymbol{M} \boldsymbol{T}^{-1}=\boldsymbol{N}, \quad \boldsymbol{N}=\left(\begin{array}{lll}
1 & 0 & 0 \\
0 & 1 & 0 \\
n & 0 & 1
\end{array}\right), \quad n=\operatorname{gcd}(\alpha, \beta)
$$

where the transformation matrix $\boldsymbol{T}$ is

$$
\boldsymbol{T}=\left(\begin{array}{ccc}
1 & 0 & 0 \\
t_{2} & 1 & 0 \\
t_{3} & t_{4} & 1
\end{array}\right)\left(\begin{array}{ccc}
s & 0 & 0 \\
0 & \beta / n & -\alpha / n \\
0 & k & l
\end{array}\right)
$$

where $s= \pm 1, k, l$ are solutions of $k \alpha+l \beta=n s$, and $t_{2}, t_{3}, t_{4}$ are arbitrary integers. This equation always has solutions (see, e.g., [24]) and for definiteness we take the one with smallest Euclidean norm for $k, l$. The choice of the position of the $n$ in $N$ is not essential but convenient; similarly, we will choose $s=1$ in the following.

The new actions $\boldsymbol{J}=\boldsymbol{T} \boldsymbol{I}$ transform by $\boldsymbol{N}$ under continuation around a loop enclosing the focus point. The second row of the second factor of $\boldsymbol{T}$ therefore determines the linear combination $J_{2}=$ $\beta I_{2} / n-\alpha I_{3} / n$ of natural actions that is invariant under $\boldsymbol{M}$. The corresponding solution is $k=0$, $l=-1$ so that $\boldsymbol{T}$ becomes upper triangular if we choose the first factor to be the identity. Non-zero entries $t_{i}$ would merely modify the new actions by linear combinations of invariant actions. Even though $\boldsymbol{T}$ is not uniquely defined, the second invariant action is unique modulo the trivial invariant action; naturally the "bad" action cannot be uniquely defined. In the prolate ellipsoid we have $\alpha=2$ and $\beta=-1$ so that the monodromy invariant actions are $I_{1}$ and $I_{2}+2 I_{3}$.

The essential ingredient for the appearance of classical monodromy in a smooth two degrees of freedom system is a focus point, i.e., an equilibrium point with complex (or at least degenerate) multipliers. To show the relation to our setting we calculate the stability of the periodic orbit corresponding to the focus line $F$. It is the orbit that is bouncing back and forth along the symmetry axis of the prolate ellipsoid. Its stability is obtained from the two matrices (see [25])

$$
S_{T}=\left(\begin{array}{cccc}
1 & 0 & l & 0 \\
0 & 1 & 0 & l \\
0 & 0 & 1 & 0 \\
0 & 0 & 0 & 1
\end{array}\right), \quad S_{R}=\left(\begin{array}{cccc}
-1 & 0 & 0 & 0 \\
0 & 1 & 0 & 0 \\
2 / R_{\|} & 0 & -1 & 0 \\
0 & -2 / R_{\perp} & 0 & 1
\end{array}\right)
$$

where $l$ is the Euclidean length between two consecutive reflections, and $R_{\|}$and $R_{\perp}$ are the radii of curvature parallel and perpendicular to the reflection plane. The monodromy matrix (in the sense of 
Floquet theory) of the period 2 orbit is given by

$$
S=S_{R} S_{T} S_{R} S_{T}
$$

with $l=2$ and identical negative radii of curvature $R_{\|}=R_{\perp}=a^{2}-1$ due to the rotational symmetry. Hence, the multipliers (i.e., the eigenvalues of $S$ ) are $\lambda, 1 / \lambda, \lambda, 1 / \lambda$ with

$$
\lambda=\frac{(1-a)^{2}}{(1+a)^{2}},
$$

so that we have degenerate hyperbolic multipliers.

\section{ENERGY SURFACES}

The essential information about an integrable system is contained in the representation of its energy surfaces in action space [26]. Because of the simple scaling property of the action variables with respect to the energy it is sufficient to consider the surface for a single energy. Figure 8 shows the energy surface $H_{\text {prol }}=1 / 2$ where $H_{\text {prol }}$ denotes the Hamiltonian Eq. (11) transformed to the action variables $\left(I_{\varphi}, I_{\eta}, I_{\xi}\right)$. In fact, the actions $\left(I_{\varphi}, I_{\eta}, I_{\xi}\right)$ fulfilling $H_{\text {prol }}=1 / 2$ are simply the scaled actions $\left(I_{1}, I_{2}, I_{3}\right)$ parameterized by $l_{\varphi}$ and $\kappa^{2}$. The vectors of frequencies

$$
\boldsymbol{\omega}=\left(\omega_{\varphi}, \omega_{\eta}, \omega_{\xi}\right)^{t}=\left(\frac{\partial H_{\mathrm{prol}}}{\partial I_{\varphi}}, \frac{\partial H_{\mathrm{prol}}}{\partial I_{\eta}}, \frac{\partial H_{\mathrm{prol}}}{\partial I_{\xi}}\right)^{t}
$$

are the normals to this surface. The energy surface is symmetric about the $\left(I_{2}, I_{3}\right)$-plane because the natural actions $I_{2}$ and $I_{3}$ are even functions of $l_{\varphi}=I_{1}$.

Most of the full phase space is obtained by attaching a 3-torus to each inner point of every energy surface. The points on the boundary correspond to the lines of the bifurcation diagram in Fig. 2 and demand special considerations. In particular on the lines $\overline{F C}$ and $\overline{F B}$ the energy surface is not differentiable, while the bifurcation diagram indicates that these are regular points of the energy momentum mapping. The fact that the energy momentum mapping is regular means that it must be

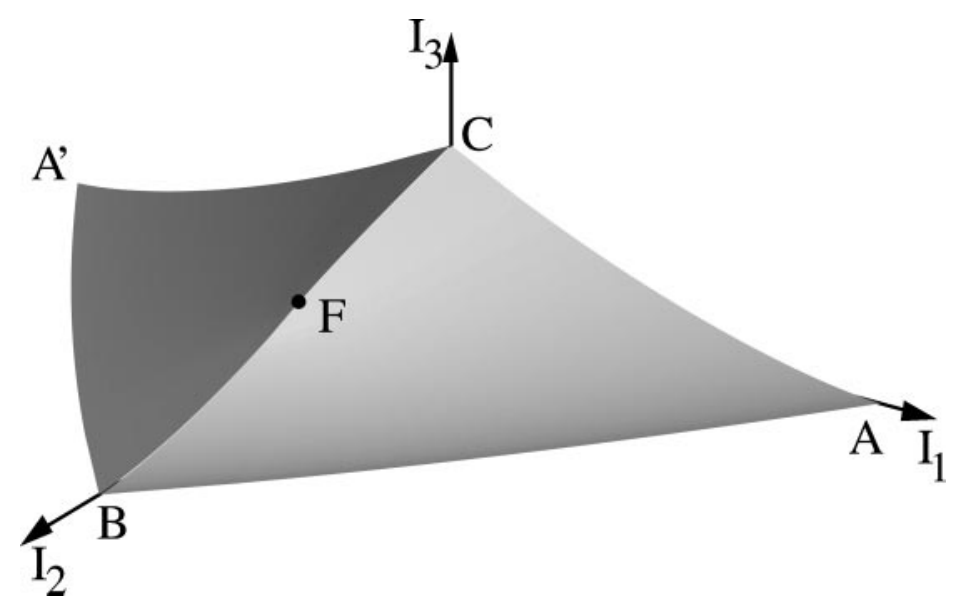

FIG. 8. Energy surface $H_{\text {prol }}(\boldsymbol{I})=1 / 2$ of the prolate ellipsoidal billiard or, equivalently, the image in $\mathbb{R}^{3}$ of the $\left(l_{\varphi}, \kappa^{2}\right)$ plane in Fig. 2 under the energy scaled actions $\left(I_{1}, I_{2}, I_{3}\right)$ from Eqs. (34)-(36). 
possible to choose actions that are smooth across this line. This was the basis of the calculation in the last section. Here we want to illustrate this calculation from a more dynamical point of view and obtain a dynamical characterization of the integers appearing in the monodromy matrix. To do this we consider three invariant two degrees of freedom subsystems contained in the three degrees of freedom billiard. They are given by (1) the billiard in the circle containing all initial conditions with $x=0, p_{x}=0 ;(2)$ the geodesic flow on the (surface of the) ellipsoid containing all initial conditions with $\xi=1, p_{\xi}=0$; and (3) the billiard in the ellipse containing initial conditions with no angular momentum about the symmetry axis, $l_{\varphi}=0$, e.g., for $z=0, p_{z}=0$.

In the first two cases we repeat the smoothing calculation in two degrees of freedom. Effectively, we look for a transformation of actions that makes the corresponding edges of the energy surface smooth. Assuming that one action $\left(l_{\varphi}\right.$ in our case) is zero at the non-smooth point we are now led to consider $2 \times 2$ matrices $M$ that have $(0, I)^{t}$ as eigenvector, hence are of the form

$$
M=\left(\begin{array}{ll}
1 & 0 \\
\mu & 1
\end{array}\right) .
$$

The frequencies $\boldsymbol{\omega}$ transform like the angles that are canonically conjugate to the actions; i.e., they transform with the inverse transpose so that

$$
\boldsymbol{\omega}_{+}=M^{-t} \boldsymbol{\omega}_{-}=M^{-t} S \boldsymbol{\omega}_{+}
$$

for $I_{1}=0$. Here $S=\operatorname{diag}(-1,1)$. This gives $2 \omega_{1}+\mu \omega_{2}=0$. The most important dynamical quantity of a 2-torus is its winding (or rotation) number $W=\omega_{1} / \omega_{2}$. The induced action of $M$ on $W$ is a Möbius transformation. The above result expressed in terms of $W$ reads

$$
\mu=-2 W
$$

We should stress again that $W$ will be the natural winding number, i.e., the one calculated in the basis of cycles obtained from the separating coordinate system and in the limit of $l_{\varphi}$ approaching zero from above.

\subsection{Circle Billiard}

Along the curves $\overline{C A}$ and $\overline{C A^{\prime}}$ we have $s_{1}^{2}=0$ so that $\kappa^{2}=l_{\varphi}^{2}$ and $I_{2}=0$. The corresponding motion is confined to the equatorial plane $x=\eta=0$. This is the billiard motion in a circle of radius $\sqrt{1-a^{2}}$. The action integral becomes elementary. One finds

$$
\begin{aligned}
& I_{\text {circ } 1} \equiv \frac{I_{\text {circ } \varphi}}{\sqrt{2 E}}=l_{\varphi}, \\
& I_{\text {circ } 3} \equiv \frac{I_{\text {circ } \xi}}{\sqrt{2 E}}=\frac{\sqrt{1-a^{2}}}{\pi}(\sin \phi-\phi|\cos \phi|)
\end{aligned}
$$

with

$$
\sin ^{2} \phi=\frac{1-s_{2}^{2}}{1-a^{2}}=1-\frac{l_{\varphi}^{2}}{1-a^{2}}
$$

The angle $\phi \in[0, \pi / 2]$ is the angle between a trajectory and the tangent to the circle at the point of its last reflection. The energy surface $H_{\operatorname{circ}}\left(I_{\operatorname{circ} \varphi}, I_{\operatorname{circ} \xi}\right)=1 / 2$ of the circle billiard is shown in Fig. 9a. It consists of two symmetric branches corresponding to the two senses of rotation in the circle billiard. Each point between $C$ and $A$ and between $C$ and $A^{\prime}$ represents a 2-torus. The points 

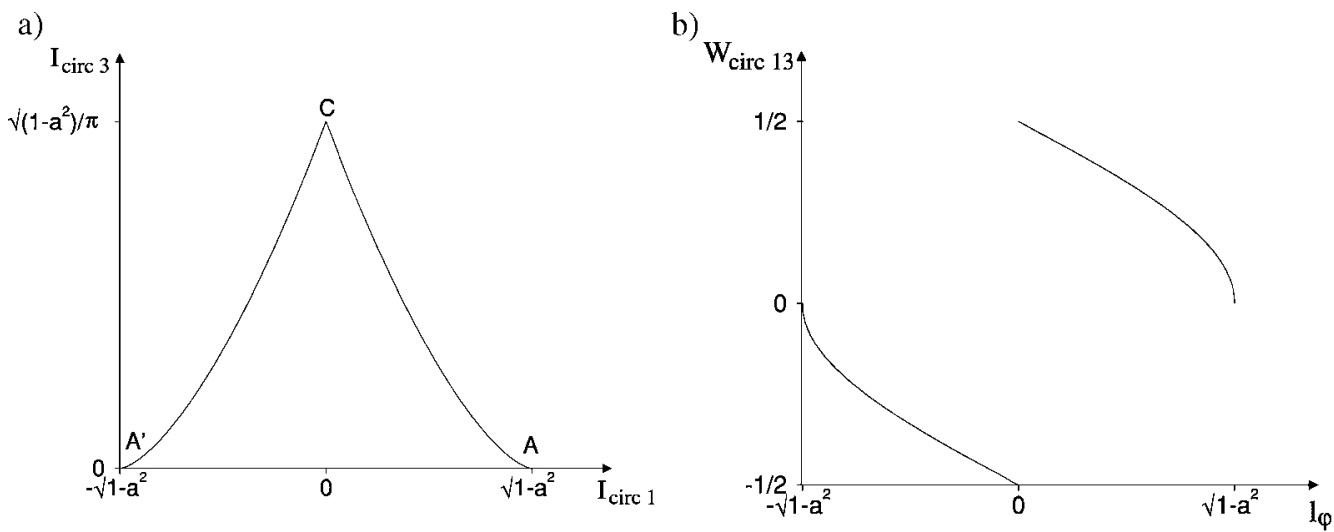

c)

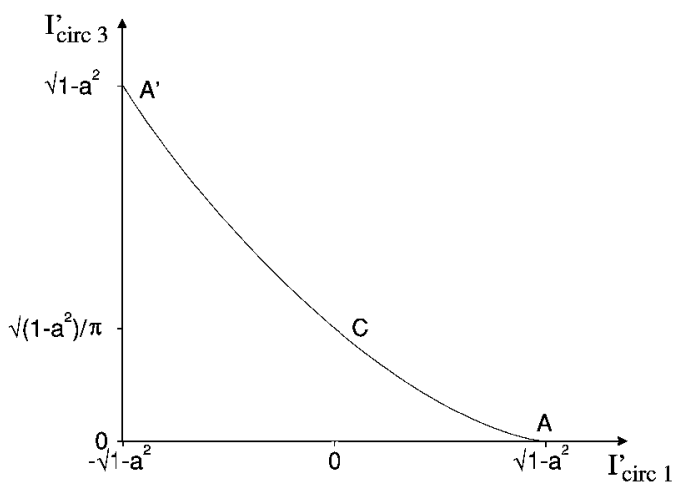

d)

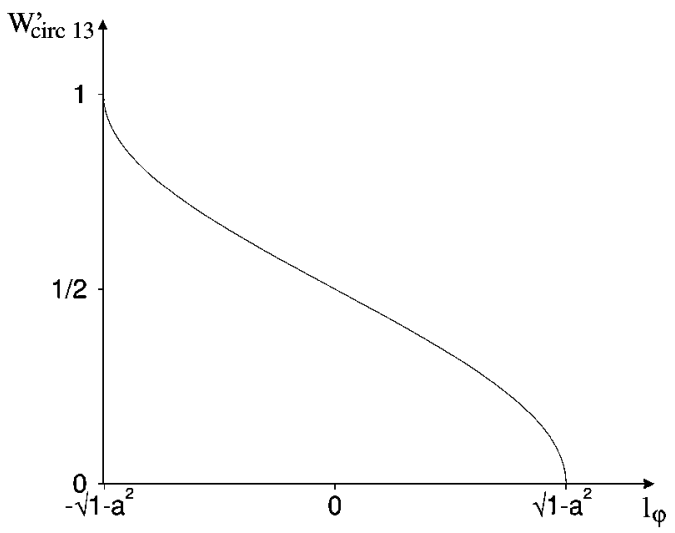

FIG. 9. Circle billiard: energy surface (a) and winding number $W_{\text {circ }} 13$ versus $l_{\varphi}$ (b). (c) and (d) are the smoothed versions of (a) and (b) as explained in the text.

$A$ and $A^{\prime}$ correspond to the two directions of the sliding motion along the billiard boundary. They are the limiting cases of the so-called whispering gallery orbits [27] which for large angular momentum evolve close to the boundary with a large number of reflections per rotation about the center of the circle. They are present in any two-dimensional billiard with a smooth strictly convex boundary [28]. The whispering gallery orbits are responsible for the energy surface to approach the $I_{1}$-axis tangentially. Point $C$ represents the periodic orbits which oscillate through the center of the circle billiard. These period-2 orbits appear as a one-parameter family parameterized by the orientation $\varphi$. They foliate a resonant 2-torus. Figure $9 \mathrm{~b}$ shows the winding number $W_{\operatorname{circ} 13}=\omega_{\operatorname{circ} \varphi} / \omega_{\operatorname{circ} \xi}$. It is the negative slope of the energy surface; i.e., the winding number becomes

$$
W_{\text {circ } 13}=-\frac{\mathrm{d} I_{\text {circ } 3}}{\mathrm{~d} l_{\varphi}}=\operatorname{sgn}\left(l_{\varphi}\right) \frac{\phi}{\pi} .
$$

$W_{\text {circ } 13}$ is an odd function of $l_{\varphi}$. For the sliding orbits it approaches zero with a diverging slope; i.e., the curvature of the energy surface diverges here. This is again a characteristic of billiard systems; see also [22]. For the period-2 orbits

$$
W_{\text {circ } 13}=1 / 2 \quad \text { or }-1 / 2
$$

depending on whether we approach $l_{\varphi}=0$ from above or from below. Accordingly, we find for 
Eq. (58), $\mu=-1$. The smooth energy surface corresponding to the new action variables

$$
\left(\begin{array}{l}
I_{\operatorname{circ} 1}^{\prime} \\
I_{\operatorname{circ} 3}^{\prime}
\end{array}\right)= \begin{cases}\left(\begin{array}{ll}
I_{\operatorname{circ} 1} \\
I_{\operatorname{circ} 3}
\end{array}\right), & l_{\varphi} \geq 0 \\
\left(\begin{array}{cc}
1 & 0 \\
-1 & 1
\end{array}\right)\left(\begin{array}{l}
I_{\operatorname{circ} 1} \\
I_{\operatorname{circ3}}
\end{array}\right), & l_{\varphi} \leq 0\end{cases}
$$

and the corresponding smooth winding number $W_{\text {circ } 13}^{\prime}$ are shown in Figs. 9c and d.

All the orbits in the circle billiard form a three-dimensional invariant submanifold within the five-dimensional energy surface of the three degrees of freedom billiard flow. The winding number in Eq. (64) characterizes the response of the 2-tori to perturbations that stay in this submanifold. If a perturbation leaves the submanifold the motion takes place on a 3-torus, hence the additional winding number $W_{23}=\omega_{\eta} / \omega_{\xi}$ evaluated for $I_{\eta}=0$ has to be considered. In general it holds that

$$
W_{23}=-\left.\frac{\partial I_{\xi}}{\partial I_{\eta}}\right|_{E, I_{\varphi}}=-\frac{\partial I_{3} / \partial \kappa^{2}}{\partial I_{2} / \partial \kappa^{2}}
$$

so that for $I_{\eta}=0$ we find

$$
W_{23}=\frac{1}{2}\left(1-\frac{2}{\pi} \chi\right), \quad \text { with } \sin ^{2} \chi=1-\left(1-a^{2}\right) \sin ^{2} \phi
$$

\subsection{Geodesic Flow}

Along the curves $\overline{B A}$ and $\overline{B A^{\prime}}$ we have $s_{2}^{2}=1$. The corresponding motion is the geodesic flow on the prolate ellipsoidal surface in Eq. (1). In this case the dynamically relevant branch point $s_{2}$ lies on the billiard boundary $\xi=1$ so that $I_{3}=0$. In terms of Legendre's standard integrals we find

$$
\begin{aligned}
& I_{\text {geod } 1} \equiv \frac{I_{\text {geod } \varphi}}{\sqrt{2 E}}=l_{\varphi}, \\
& I_{\text {geod } 2} \equiv \frac{I_{\text {geod } \eta}}{\sqrt{2 E}}=\frac{2}{\pi}\left(\mathcal{E}\left(s_{1}\right)-\left(1-s_{1}^{2}\right) \Pi\left(-\beta^{2}, s_{1}\right)\right), \quad \beta^{2}=s_{1}^{2} \frac{1-a^{2}}{a^{2}-s_{1}^{2}} .
\end{aligned}
$$

Note that the appearance of an integral of third kind for the geodesic flow is due to the fact that we consider the degenerate rotationally symmetric version of Jacobi's problem of the geodesic flow on general triaxial ellipsoids $[29,30]$. The energy surface $H_{\operatorname{geod}}\left(I_{\operatorname{geod} \varphi}, I_{\operatorname{geod} \eta}\right)=1 / 2$ is shown in Fig. 10a. Again it consists of two symmetric branches corresponding to the two senses of rotation of the coordinate $\varphi$. The points between $B$ and $A$ and between $B$ and $A^{\prime}$ again represent 2-tori. The circle motions at $A$ and $A^{\prime}$ appear by collapsing the two $\eta$-caustics $\eta= \pm s_{1}$ for $s_{1}^{2} \rightarrow 0$. Point $B$ represents planar periodic orbits sliding along ellipses. These periodic orbits may be parameterized by the orientation $\varphi$ and like the periodic orbits at point $C$ of the circle billiard they foliate a resonant 2 -torus. The different senses of rotation are related by the angles $\varphi$ and $\varphi+\pi$. The negative slope of the energy surface is the winding number

$$
\begin{aligned}
W_{\operatorname{geod} 12} & =\frac{\omega_{\operatorname{geod} \varphi}}{\omega_{\operatorname{geod} \eta}}=-\frac{\mathrm{d} I_{\operatorname{geod} 2}}{\mathrm{~d} l_{\varphi}} \\
& =\operatorname{sgn}\left(l_{\varphi}\right) \frac{2 a\left(2-\kappa^{2}-a^{2}\right)}{\pi \sqrt{\left(1-a^{2}\right)\left(1-\kappa^{2}\right)}} \Pi\left(-\beta^{2}, k\right),
\end{aligned}
$$


a)

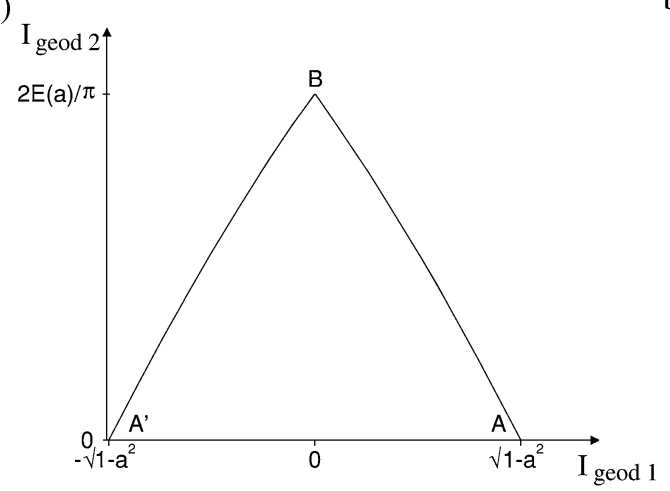

c)

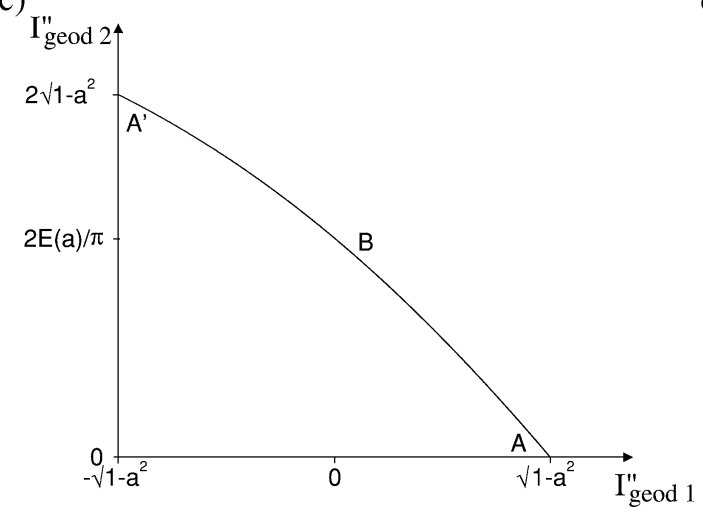

b)

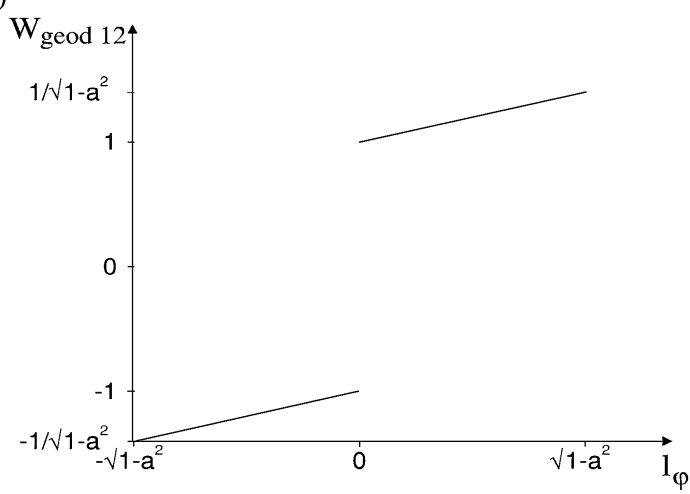

d)

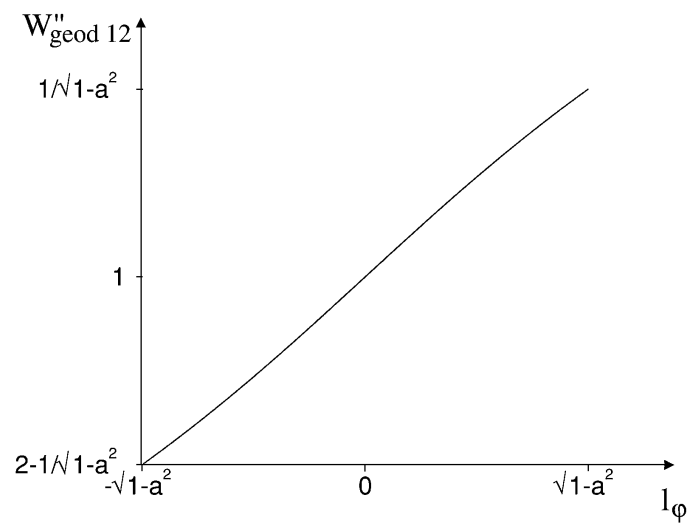

FIG. 10. Geodesic flow: energy surface (a) and winding number $W_{\text {geod } 12}$ versus $l_{\varphi}$ (b). (c) and (d) are the smoothed versions of (a) and (b) as explained in the text.

which is shown in Fig. 10b. The limiting value for positive $l_{\varphi}$ is 1 , so that $\mu=-2$ in this case. The smooth energy surface corresponding to the new action variables

$$
\left(\begin{array}{l}
I_{\operatorname{geod} 1}^{\prime \prime} \\
I_{\operatorname{geod} 2}^{\prime \prime}
\end{array}\right)= \begin{cases}\left(\begin{array}{l}
I_{\operatorname{geod} 1} \\
I_{\operatorname{geod} 2}
\end{array}\right), & l_{\varphi} \geq 0 \\
\left(\begin{array}{cc}
1 & 0 \\
-2 & 1
\end{array}\right)\left(\begin{array}{l}
I_{\operatorname{geod} 1} \\
I_{\operatorname{geod} 2}
\end{array}\right), & l_{\varphi} \leq 0\end{cases}
$$

and the new winding number $W_{\text {geod } 12}^{\prime \prime}$ are shown in Figs. 10c and d.

The geodesic flow is the limiting case of the whispering gallery orbits in the three-dimensional prolate ellipsoidal billiard. Again there is a third frequency characterizing perturbations that leave the geodesic subflow. For them the frequency $\omega_{\xi}$ goes to infinity in the geodesic limiting case, so that $W_{13}=0$.

\subsection{Ellipse Billiard}

Along the lines $\overline{F C}$ and $\overline{F B}$ we have $l_{\varphi}=0$, so that the motion takes place in a plane containing the symmetry axis; i.e., it is just the ordinary billiard in the ellipse. We already stressed that these lines in the bifurcation diagram (except for $\kappa^{2}=0, a^{2}, 1$ ) are not critical values of the energy momentum 
a)

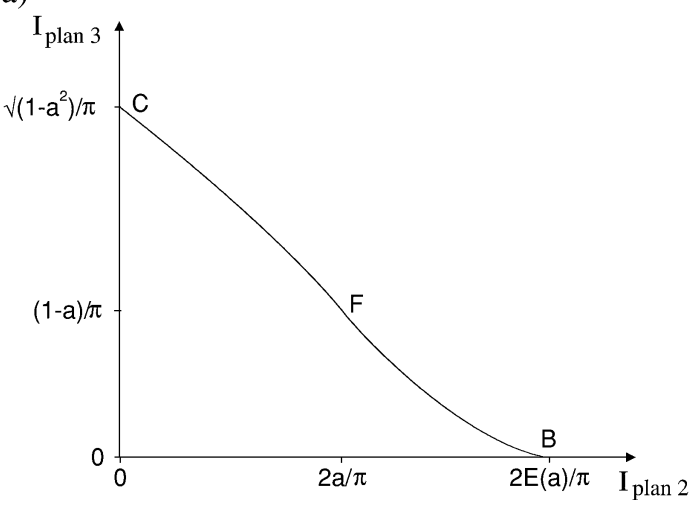

b)

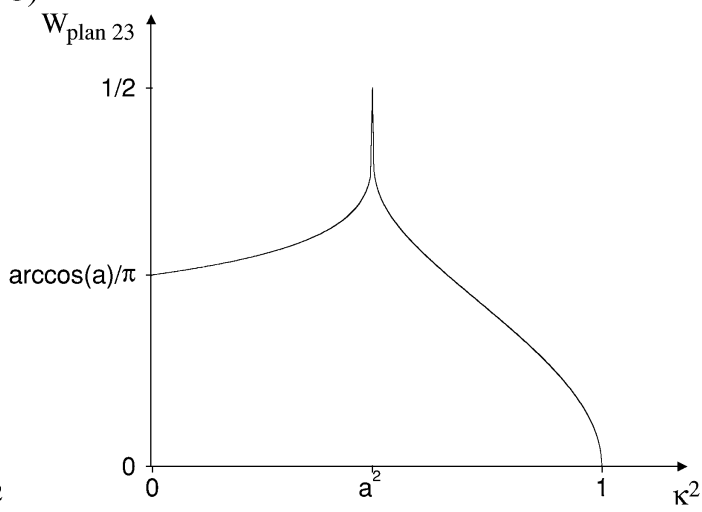

FIG. 11. Ellipse billiard: energy surface (a) and winding number $W_{\mathrm{plan} \xi \eta}$ against $\kappa^{2}$ (b).

mapping. Therefore their preimages in phase space are 3-tori. However, from the dynamical point of view these 3-tori are special because they are all resonant.

Along $\overline{F B}$ the action integrals $I_{2}$ and $I_{3}$ reduce to

$$
\begin{aligned}
& I_{\text {plan } 2} \equiv \frac{I_{\text {plan } \eta}}{\sqrt{2 E}}=\frac{2 s_{2}}{a} \mathcal{E}(k), \\
& I_{\text {plan } 3} \equiv \frac{I_{\text {plan } \xi}}{\sqrt{2 E}}=\frac{1}{\pi}\left(\sin \chi-s_{2} \mathcal{E}(\chi, k)\right)
\end{aligned}
$$

with $k$ and $\chi$ from Eq. (39) with $s_{1}$ replaced by $a$. Along $\overline{F C}$ they become

$$
\begin{aligned}
& I_{\text {plan } 2} \equiv \frac{I_{\text {plan } \eta}}{\sqrt{2 E}}=\frac{2}{\pi}\left(a \mathcal{E}(k)-\frac{a^{2}-s_{1}^{2}}{a} \mathcal{K}(k)\right), \\
& I_{\text {plan } 3} \equiv \frac{I_{\text {plan } \xi}}{\sqrt{2 E}}=\frac{1}{\pi}\left(\sin \chi+\frac{a^{2}-s_{1}^{2}}{a} \mathcal{F}(\chi, k)-a \mathcal{E}(\chi, k)\right),
\end{aligned}
$$

with $s_{2}$ replaced by $a$ in $k$ and $\chi$. The actions $\left(I_{\text {plan } 2}, I_{\text {plan } 3}\right)$ correspond to the edge $I_{1}=0$ of the energy surface of the full billiard flow which we represent once more in Fig. 11a. Up to some trivial factors 2 it is the energy surface of the elliptic billiard in [10]. The two cases can be identified with the two types of motion in the ellipse billiard; see Fig. 12. On $\overline{F B}$ the caustic is around the two foci of the ellipse, while on $\overline{F C}$ the caustic is between the foci. The negative slope of the energy surface

a)

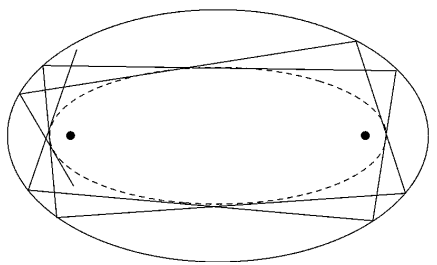

b)

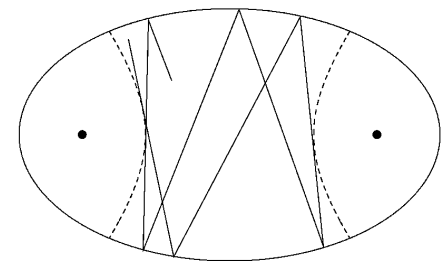

FIG. 12. Generic motions in the ellipse billiard: whispering gallery orbit (a) and bouncing ball orbit (b). 
in Fig. 11a is the winding number $W_{\text {plan } \eta \xi}=\omega_{\text {plan } \eta} / \omega_{\text {plan } \xi}$ for which one finds

$$
W_{\text {plan } 23}=-\left.\frac{\partial I_{\text {plan } \xi}}{\partial I_{\operatorname{plan} \eta}}\right|_{E}= \begin{cases}-\left(\frac{\mathrm{d} I_{\text {plan } 3}}{\mathrm{~d} s_{2}}\right) /\left(\frac{\mathrm{d} I_{\text {plan } 2}}{\mathrm{~d} s_{2}}\right)=\frac{1}{2} \frac{\mathcal{F}(\chi, k)}{\mathcal{K}(k)} & \text { (line } \overline{F B}) \\ -\left(\frac{\mathrm{d} I_{\text {plan } 3}}{\mathrm{~d} s_{1}}\right) /\left(\frac{\mathrm{d} I_{\text {plan } 2}}{\mathrm{~d} s_{1}}\right)=\frac{1}{2} \frac{\mathcal{F}(\chi, k)}{\mathcal{K}(k)} & \text { (line } \overline{F B})\end{cases}
$$

see Fig. $11 \mathrm{~b}$. At the focus point $F$ the winding number $W_{\text {plan } 23}$ displays a cusp or, to be precise, it continuously assumes the value $1 / 2$ for $\kappa^{2} \rightarrow a^{2}$ but the derivative diverges in this limit.

In order to find the winding numbers characterizing orbits that leave the ellipse subflow let us consider again the relation in Eq. (59) but now considered in the full three degrees of freedom system restricted to $I_{1}=l_{\varphi}=0$. Inserting the $3 \times 3$ matrices $\boldsymbol{M}_{i}$ gives

$$
2 \omega_{\varphi}+\alpha_{i} \omega_{\eta}+\beta_{i} \omega_{\xi}=0
$$

As before we have $\alpha_{1}=0$ for $\kappa^{2}<a^{2}$ and $\beta_{2}=0$ for $\kappa^{2}>a^{2}$. Therefore, one of the two winding numbers of the full system is constant on the line $l_{\varphi}=0$ :

$$
\begin{array}{rr}
W_{\text {plan 13 }}=\frac{\omega_{\varphi}}{\omega_{\xi}}=-\beta_{1} / 2=1 / 2 & \text { for } \left.\kappa^{2}<a^{2} \text { (line } \overline{F C}\right), \\
W_{\text {plan } 12}=\frac{\omega_{\varphi}}{\omega_{\eta}}=-\alpha_{2} / 2=1 & \text { for } \left.\kappa^{2}>a^{2} \text { (line } \overline{F B}\right) .
\end{array}
$$

The interesting winding number from the point of view of monodromy is not the one of the ellipse billiard $W_{\text {plan 23 }}$, but the ones that describe the perturbations away from it. Due to the symmetry of the prolate ellipsoid there is no rotational motion at all if we start with $l_{\varphi}=0$. The generic motion in the ellipse billiard is on a 2-torus, and rotating it around the symmetry axis generates the 3-torus of the full system. The fact that it is foliated into invariant 2-tori is due to the resonances (79). When we loop around the focus point $F$ in order to determine the monodromy the main effect is picked up upon crossing these resonant tori of the ellipse billiard.

\subsection{Smooth Energy Surfaces}

Before we proceed let us remark that the previous sections have shown that the natural actions $I_{2}$ and $I_{3}$ have the nice property of being invariant under the system's discrete symmetry of time reversal combined with $l_{\varphi} \mapsto-l_{\varphi}$. This means that these actions are also "natural" from a dynamical point of view. Nevertheless, we now want to make the actions as smooth as possible, at the expense of breaking this invariance.

In order to smooth the energy surface of the three degrees of freedom billiard flow in Fig. 8 we have to apply the matrices $\boldsymbol{M}_{1}$ and $\boldsymbol{M}_{2}$ with the $\alpha_{i}$ and $\beta_{i}$ defined in Eqs. (49) and (50). Note that these matrices are just the three-dimensional generalizations of the $2 \times 2$ matrices defined to smooth the actions of the circle billiard and the geodesic flow according to Eqs. (66) and (73), respectively. This means that the boundary of the energy surface alone determines the smooth actions of the full three degrees of freedom system. However, the application of the matrices $\boldsymbol{M}_{1}$ and $\boldsymbol{M}_{2}$ does not give a globally smooth energy surface-only half of its edge is removed by either transformation; see Figs. 13 and 14. The impossibility of finding a globally smooth and single valued set of actions is one indicator of classical monodromy.

We have already illustrated the importance of frequencies and winding numbers in our analysis of the two-dimensional invariant subsystems. Now we are going to consider them for the full system. For two degrees of freedom this is fairly simple and the smoothed winding numbers are shown in Figs. 9d and 10d. For more degrees of freedom it is most appropriate to think about the frequency 


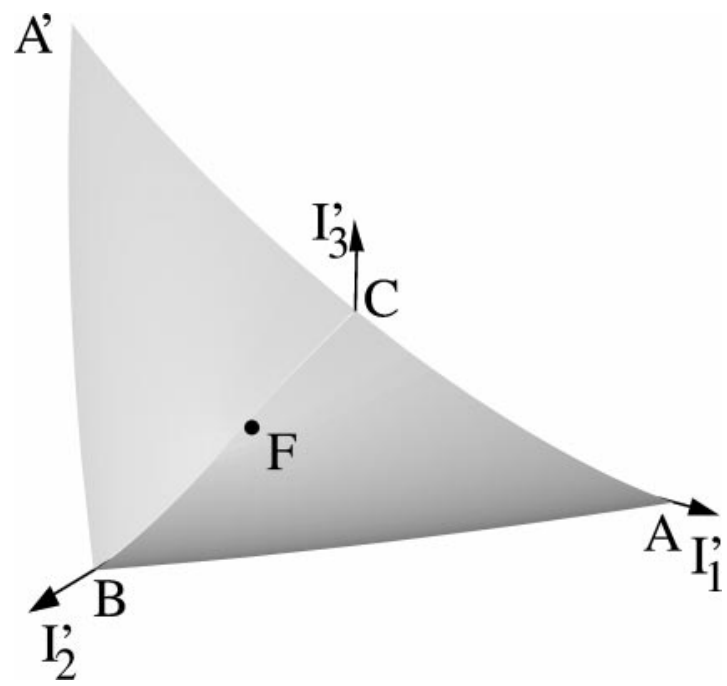

FIG. 13. Partially smoothed energy surface of the prolate ellipsoidal billiard. The $\boldsymbol{I}^{\prime}$ are the natural actions transformed with the unimodular matrix $\boldsymbol{M}_{1}$ for $l_{\varphi} \leq 0$.

ratios in terms of the Gauss map. The Gauss map is the map from a hypersurface of $\mathbb{R}^{n}$ to the unit sphere $S^{n-1}$. Each point of the hypersurface is mapped to the normalized normal vector in $S^{n-1}$ at that point. In our setting the Gauss map maps the two-dimensional energy surface in action space to the unit sphere $S^{2}$ of the normalized frequencies $\boldsymbol{\omega}$. The determinant of the Jacobian of the Gauss map is the curvature of the energy surface. Since the frequency ratios do not change if the frequencies are all multiplied by -1 the space of frequency ratios is the real projective space $\mathbb{R} P^{2}=S^{2} /\{ \pm\}$. The frequencies $\boldsymbol{\omega}$ can be viewed as homogeneous coordinates in the projective space, and by dehomogenizing we can obtain the winding numbers

$$
W_{12}=\frac{\omega_{\varphi}}{\omega_{\eta}}, \quad W_{23}=\frac{\omega_{\eta}}{\omega_{\xi}}
$$

as local coordinates.

In practice, we represent the projective space of the frequency ratios of the prolate ellipsoidal billiard by the half-sphere

$$
\mathcal{S}_{\mathrm{h}}=\left\{\overline{\boldsymbol{\omega}} \in \mathbb{R}^{3}:\|\overline{\boldsymbol{\omega}}\|_{2}=1, \bar{\omega}_{3} \geq 0\right\},
$$

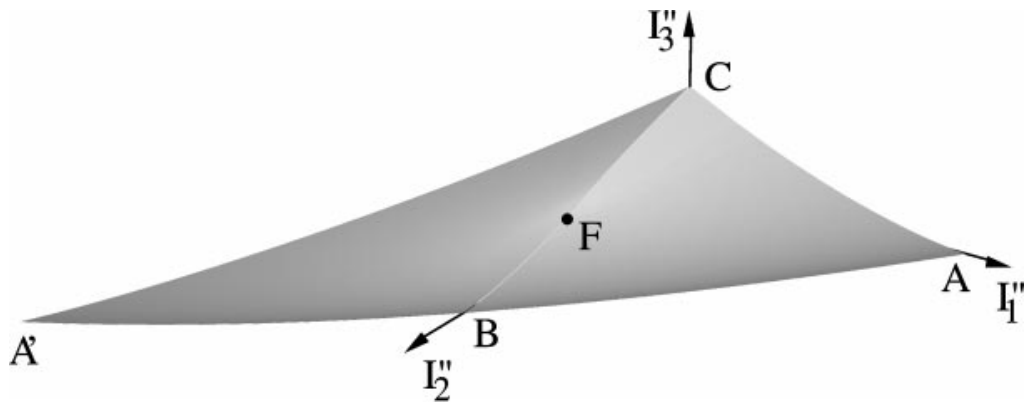

FIG. 14. Partially smoothed energy surface of the prolate ellipsoidal billiard. The $\boldsymbol{I}^{\prime \prime}$ are the natural actions transformed with the unimodular matrix $\boldsymbol{M}_{2}$ for $l_{\varphi} \leq 0$. 
where $\left(\bar{\omega}_{1}, \bar{\omega}_{2}, \bar{\omega}_{3}\right)$ are the normalized frequencies $\left(\omega_{\varphi}, \omega_{\eta}, \omega_{\xi}\right)$ from Eq. (57). On the half-sphere $\mathcal{S}_{\mathrm{h}}$ most points have a unique representant.

On $\mathcal{S}_{\mathrm{h}}$ all frequencies $\bar{\omega}$ that satisfy a given resonance condition

$$
(\omega, \boldsymbol{m})=0
$$

with a resonance vector $\boldsymbol{m} \in \mathbb{Z}$ of coprime integers are given by the great circle contained in the plane through the origin perpendicular to $\boldsymbol{m}$. Completely resonant tori satisfy two independent resonance conditions. The corresponding torus is foliated into periodic orbits. The frequency direction that satisfies two given resonance conditions $\boldsymbol{m}_{1}$ and $\boldsymbol{m}_{2}$ is given by the intersection of the corresponding great circles. It is therefore given by the cross product $\overline{\boldsymbol{m}_{1} \times \boldsymbol{m}_{2}}$. We call a direction $\boldsymbol{m}=\boldsymbol{m}_{1} \times \boldsymbol{m}_{2} \in \mathbb{Z}^{3}$ full resonance.

In Fig. 15 full resonances are indicated as dots on the sphere. The impressive pattern of the full resonances is the same for every integrable system with three degrees of freedom. The only thing which does depend on the system is the curvature and which frequency directions are possible, i.e., the range of the Gauss map.

The image of the frequencies of the prolate ellipsoidal billiard on $\mathcal{S}_{\mathrm{h}}$ give two patches according to the two signs of $\omega_{\varphi}$. The patch for $\omega_{\varphi} \geq 0$ is shown in Fig. 15a and denoted by 0 . To find its boundaries we study the images of the boundaries of the energy surface which correspond to the invariant subsystems with two degrees of freedom discussed in the last section. We use the same labeling as for the corresponding points on the energy surface. From two sides it is bounded by the great circles given by the constant winding numbers $W_{\text {plan } 13}$ and $W_{\text {plan } 12}$ of Eqs. (80) and (81),

$$
\begin{aligned}
2 \bar{\omega}_{1}-\bar{\omega}_{3} & =0, & & (\overline{F C}), \\
\bar{\omega}_{1}-\bar{\omega}_{2} & =0, & & (\overline{F B}) .
\end{aligned}
$$

The coordinates of the focus point $F$ on $\mathcal{S}_{\mathrm{h}}$ are given by the intersection of these great circles, i.e., by the cross product of the corresponding resonance vectors

$$
\overline{(1,-1,0)^{t} \times(2,0,-1)^{t}}=\overline{(1,1,2)^{t}} .
$$

Due to the divergence of the frequency component $\omega_{\xi}$ in the limiting case of the geodesic flow the complete edge $\overline{A B}$ of the energy surface is mapped to the single point $\bar{\omega}=(0,0,1)^{t}$. The curve $\overline{A C}$ connecting this point and $C$ on $\mathcal{S}_{\mathrm{h}}$ results from the circle billiard winding numbers as the cross product of the lines

$$
\begin{aligned}
& \omega_{1}-W_{\text {circ } 13} \omega_{3}=0, \\
& \omega_{1}-W_{\text {circ } 12} \omega_{2}=0 ;
\end{aligned}
$$

i.e., $\overline{A C}$ is parameterized by

$$
\overline{\left(1,0,-\frac{\phi}{\pi}\right)^{t} \times\left(0,1, \frac{\chi}{\pi}-\frac{1}{2}\right)^{t}}=\overline{\left(\frac{\phi}{\pi}, \frac{1}{2}-\frac{\chi}{\pi}, 1\right)^{t}}
$$

with $\phi$ and $\chi$ from Eqs. (63) and (68).

Applying the transformations $\boldsymbol{M}_{1}$ or $\boldsymbol{M}_{2}$ to the actions induces the transformations $\boldsymbol{M}_{i}^{t^{-1}}$ on the frequencies $\boldsymbol{\omega}_{-}$. In Fig. 15a the corresponding patches are labeled by the indices 1 and 2 . They again are triangularly shaped. The partial smoothness of the energy surfaces in Figs. 13 and 14 is reflected by the fact that the patches 1 and 2 have one boundary line in common with patch $0: \overline{C F}$ for patch 1 
a)

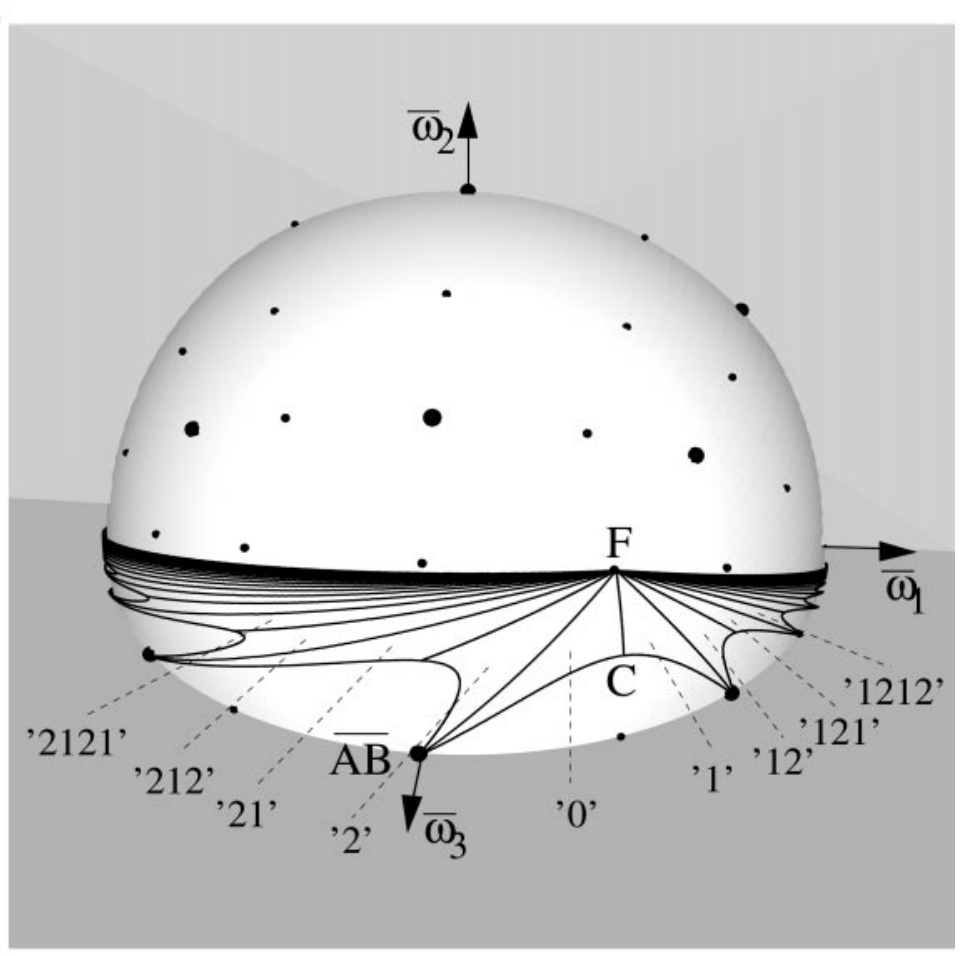

b)

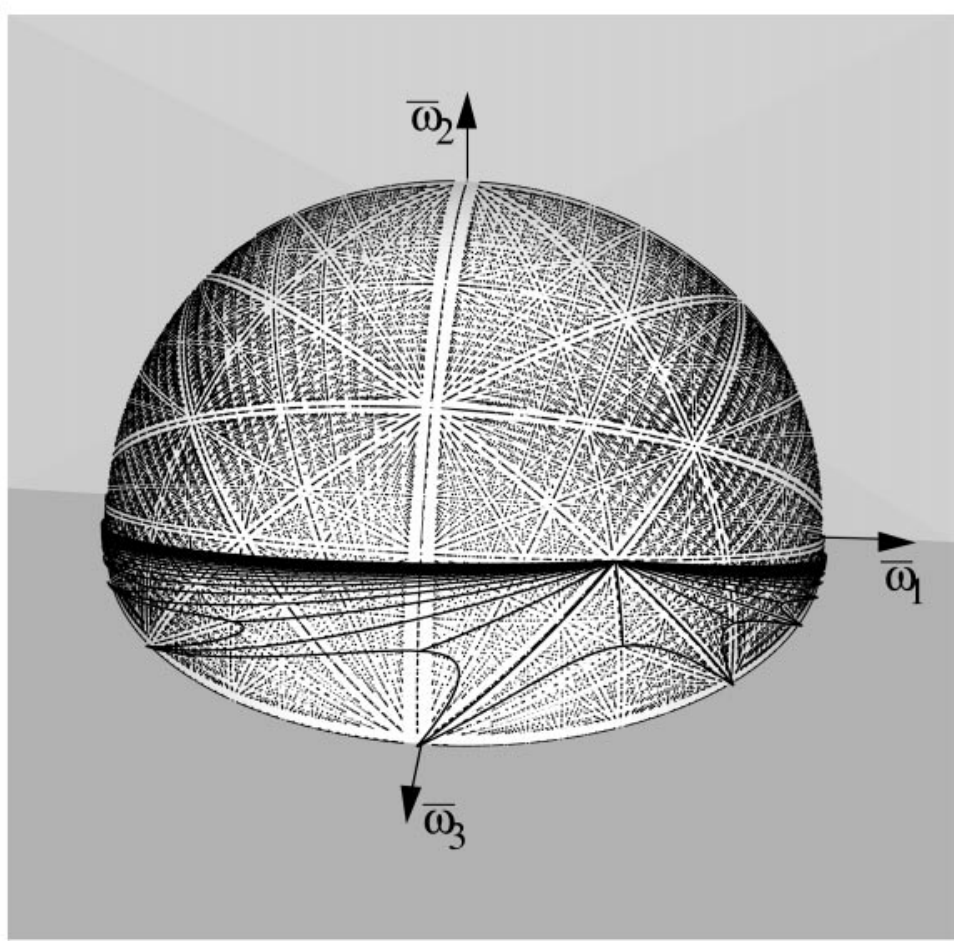

FIG. 15. (a) Half the frequency half-sphere $\mathcal{S}_{\mathrm{h}}$ with frequency patches of the prolate ellipsoidal billiard with full resonances $\|\boldsymbol{m}\|_{\infty}=\max \left\{\left|m_{1}\right|,\left|m_{2}\right|,\left|m_{3}\right|\right\}=1$ (big dots) and $\|\boldsymbol{m}\|_{\infty}=2$ (small dots). Patch 0 represents the frequencies in Eq. (57) for $l_{\varphi} \geq 0$. Patch ${ }^{\prime} i_{1} i_{2} \ldots i_{n}^{\prime}$ corresponds to $\left\{\hat{\boldsymbol{M}}_{i_{1}}^{t^{-1}} \hat{\boldsymbol{M}}_{i_{2}}^{t^{-1}} \cdots \hat{\boldsymbol{M}}_{i_{n}}^{t^{-1}} \boldsymbol{\omega}: \omega_{1} \geq 0\right\}$ where $i_{1}, i_{2}, \ldots, i_{n} \in\{1,2\}$. (b) Same as (a) now with full resonances $\|\boldsymbol{m}\|_{\infty} \leq 30$. 


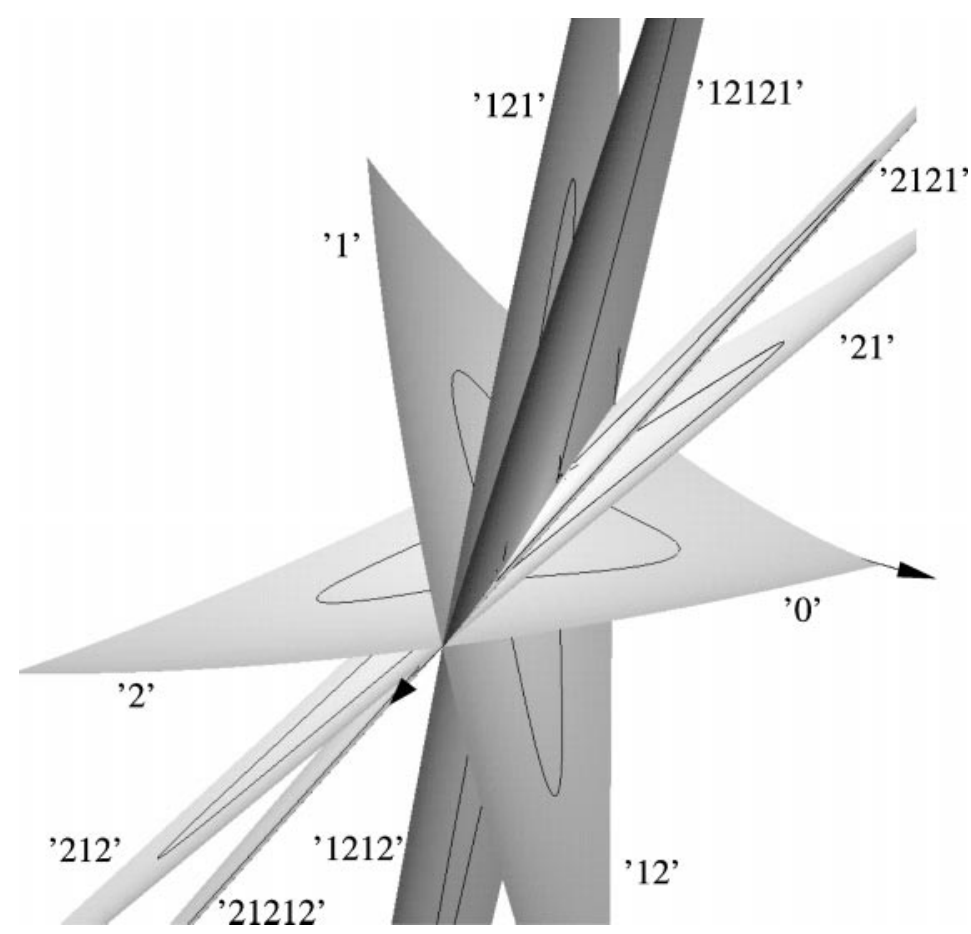

FIG. 16. Patch 0 represents the energy surface for action variables $\left(I_{1}, I_{2}, I_{3}\right)$ in Eqs. (34)-(36) with $l_{\varphi} \geq 0$. Patch $i_{1} i_{2} \ldots i_{n}$ corresponds to $\left\{\hat{\boldsymbol{M}}_{i_{1}} \hat{\boldsymbol{M}}_{i_{2}} \ldots \hat{\boldsymbol{M}}_{i_{n}} \boldsymbol{I}\left(l_{\varphi}, \kappa^{2}\right): l_{\varphi} \geq 0\right\}$ where $i_{1}, i_{2}, \ldots, i_{n} \in\{1,2\}$.

and $\overline{A F}$ for patch 2 . The common boundary line says that the frequency map is continuous across the boundary; in our case it actually is analytic.

Figures 13 and 14 suggest continuing the smoothing procedure to the remaining edges $\overline{C F}$ and $\overline{B F}$, respectively. This indeed can be achieved by successively applying the transformations $\hat{\boldsymbol{M}}_{i}=\boldsymbol{M}_{i} \boldsymbol{S}$. We do not have to take the inverse because $\hat{\boldsymbol{M}}_{i}=\hat{\boldsymbol{M}}_{i}^{-1}$. In this way the rosette type of energy surface in Fig. 16 is obtained. Away from $F$ this surface is everywhere smooth. The smoothly continued action defined this way is a multivalued function of $l_{\varphi}$ and $\kappa^{2}$ where the different leaves are related by powers of the monodromy matrix $\boldsymbol{M}$.

The smoothness of the surface Fig. 16 becomes apparent from the representation of its frequencies which are shown in Fig. 15. The analogue of the monodromy matrix $\boldsymbol{M}$ in frequency space is

$$
\boldsymbol{M}_{\omega}=\boldsymbol{M}^{t^{-1}}=\left(\begin{array}{ccc}
1 & -2 & 1 \\
0 & 1 & 0 \\
0 & 0 & 1
\end{array}\right) .
$$

$\boldsymbol{M}_{\omega}$ has eigenvalue 1 with multiplicity 3 and eigenspace $\operatorname{span}\left\{(0,1,2)^{t},(1,0,0)^{t}\right\}$. The cross product

$$
(0,1,2)^{t} \times(1,0,0)^{t}=(0,2,-1)^{t}
$$

gives the resonance that defines the "limiting great circle" on $\mathcal{S}_{\mathrm{h}}$ in Figs. 15a and 15b. Points on the limiting great circle are invariant under $\boldsymbol{M}_{\omega}$. 


\section{QUANTUM MONODROMY}

The implications of the classical monodromy on the quantum mechanical spectrum can be understood in terms of the EBK quantization of actions. In the space of natural actions the quantum spectrum defines a regular lattice. Due to the multivaluedness of smooth actions the regular lattice in action space transforms to a lattice in the space of the smooth constants of motion with a non-trivial global topology.

\subsection{The Quantum System}

The quantum mechanical billiard problem is described by Schrödinger's equation for a freely moving particle with a vanishing wave amplitude on the billiard boundary. For the prolate ellipsoidal billiard this means determining the spectrum of the three-dimensional Helmholtz's equation

$$
-\frac{\hbar^{2}}{2} \nabla^{2} \psi=E \psi
$$

for Dirichlet boundary conditions on the billiard boundary in Eq. (1). Similarly to the classical problem, this boundary value problem is separable in the coordinates $(\varphi, \eta, \xi)$.

The differential equation for $\varphi$ immediately separates off and gives the eigenvalue equation for the angular momentum operator about the $x$-axis $\hat{L}_{x}$ with spectrum

$$
L_{x}=n_{\varphi} \hbar, \quad n_{\varphi} \in \mathbb{Z}
$$

Using (94) the Hamilton operator $\hat{H}=-\frac{\hbar^{2}}{2} \nabla^{2}$ becomes

$$
\hat{H}=-\frac{\hbar^{2}}{2} \frac{1}{\xi^{2}-\eta^{2}}\left(\frac{\partial}{\partial \xi}\left(\xi^{2}-a^{2}\right) \frac{\partial}{\partial \xi}+\frac{\partial}{\partial \eta}\left(a^{2}-\eta^{2}\right) \frac{\partial}{\partial \eta}\right)+\frac{a^{2}}{2} \frac{\hbar^{2} n_{\varphi}^{2}}{\left(\xi^{2}-a^{2}\right)\left(\eta^{2}-a^{2}\right)} .
$$

For a solution $\hat{H} \psi(\eta, \xi)=E \psi(\eta, \xi)$ the ansatz $\psi(\eta, \xi)=\psi_{\eta}(\eta) \psi_{\xi}(\xi)$ leads to the eigenvalue equations

$$
\begin{aligned}
& \hat{K} \psi_{\eta} \equiv\left[-\frac{\hbar^{2}}{2} \frac{\partial}{\partial \eta}\left(a^{2}-\eta^{2}\right) \frac{\partial}{\partial \eta}+\frac{a^{2}}{2} \frac{\hbar^{2} n_{\varphi}^{2}}{a^{2}-\eta^{2}}+E \eta^{2}\right] \psi_{\eta}=K \psi_{\eta}, \\
& \hat{K} \psi_{\xi} \equiv\left[\frac{\hbar^{2}}{2} \frac{\partial}{\partial \xi}\left(\xi^{2}-a^{2}\right) \frac{\partial}{\partial \xi}-\frac{a^{2}}{2} \frac{\hbar^{2} n_{\varphi}^{2}}{\xi^{2}-a^{2}}+E \xi^{2}\right] \psi_{\xi}=K \psi_{\xi} .
\end{aligned}
$$

The operator $\hat{K}$ is the analogue of the classical separation constant $K$. In Euclidean coordinates it reads

$$
\hat{K}=\hat{\boldsymbol{L}}^{2}+a^{2} \hat{p}_{x}^{2}
$$

with the usual definition of the operators $\hat{\boldsymbol{L}}^{2}$ and $\hat{p}_{x}$ [31]. On the Hilbert space of functions that vanish on the ellipsoidal boundary the operators $\hat{H}, \hat{L}_{x}$, and $\hat{K}$ are three mutually commuting observables. The correspondence principle $-i \hbar(\partial / \partial \varphi, \partial / \partial \eta, \partial / \partial \xi) \rightarrow\left(p_{\varphi}, p_{\eta}, p_{\xi}\right)$ yields their classical pendants $H, K$, and $L_{x}$ discussed in the previous sections.

For a given $n_{\varphi}$ the exact quantum mechanical spectrum is determined through the solution of the coupled eigenvalue equations (96) and (97) which are known as the spheriodal wave equations; see [32]. The equations are identical but have to be solved on different intervals. This is the wave 
mechanical manifestation of the fact that the integrands in $I_{2}$ and $I_{3}$ in Eqs. (35) and (36) only differ in the sign. A pair $(E, K)$ is an eigenvalue if it leads to a solution $\psi$ which is regular at the regular singular points $\pm a$ and fulfills the Dirichlet boundary condition [20]. This problem can be solved numerically by a shooting method. The treatment of the billiard in the ellipse in [10] directly applies to the present case, because the spheroidal wave equation with "quantum number" $n_{\varphi}= \pm 1 / 2$ is equivalent to the Mathieu equation.

It is to be expected that the spectrum of the three quantum operators can be well approximated by the EBK quantization of the classical actions:

$$
\begin{aligned}
& I_{\varphi}\left(\left(l_{\varphi}, \kappa^{2}, E\right)_{\left(n_{\varphi}, n_{\eta}, n_{\xi}\right)}\right)=\hbar\left(n_{\varphi}+\frac{\alpha_{\varphi}}{4}\right), \\
& I_{\eta}\left(\left(l_{\varphi}, \kappa^{2}, E\right)_{\left(n_{\varphi}, n_{\eta}, n_{\xi}\right)}\right)=\hbar\left(n_{\eta}+\frac{\alpha_{\eta}}{4}\right), \\
& I_{\xi}\left(\left(l_{\varphi}, \kappa^{2}, E\right)_{\left(n_{\varphi}, n_{\eta}, n_{\xi}\right)}\right)=\hbar\left(n_{\xi}+\frac{\alpha_{\xi}}{4}\right) .
\end{aligned}
$$

Here $\boldsymbol{\alpha}=\left(\alpha_{\varphi}, \alpha_{\eta}, \alpha_{\xi}\right)^{t}$ is the vector of Maslov indices characterizing the topology of the classical 3 -torus specified by the eigenvalues $\left(l_{\varphi}, \kappa^{2}, E\right)_{\left(n_{\varphi}, n_{\eta}, n_{\xi}\right)}$. The Maslov indices depend on the choice of fundamental paths on the 3-tori. With the choice of the natural action variables $\left(I_{\varphi}, I_{\eta}, I_{\xi}\right)$ for the EBK quantization the three fundamental paths are $\left(C_{1}, C_{2}, C_{3}\right)$ of Eq. (33). The $\varphi$ degree of freedom is of rotational type giving $\alpha_{\varphi}=0$. For the determination of $\alpha_{\eta}$ and $\alpha_{\xi}$ we refer to the phase portraits in Fig. 4. The $\eta$-motion is a usual oscillation, i.e., $\alpha_{\eta}=2$. $\xi$ oscillates between a usual turning point and the reflection at the billiard boundary $\xi=1$ which wave mechanically imposes Dirichlet boundary conditions, so that $\alpha_{\xi}=3$. In the space of the actions $\left(I_{\varphi}, I_{\eta}, I_{\xi}\right)$ the semiclassical eigenvalues are thus located on the simple lattice

$$
\mathcal{L}_{\mathrm{EBK}}=\left\{\hbar\left(n_{\varphi}, n_{\eta}+\frac{1}{2}, n_{\xi}+\frac{3}{4}\right): n_{\eta}, n_{\xi}, \pm n_{\varphi} \in \mathbb{N}_{0}\right\} .
$$

The transformation of the exact eigenvalues to action space shows that they are very close to the lattice points of $\mathcal{L}_{E B K}$. Instead of a three-dimensional plot we restrict ourselves to the presentation of the eigenvalues in the planes $I_{\varphi}=0$ and $I_{\varphi}=1 \hbar$; see Figs. 17 and 18 . The only noticeable deviations between the exact quantization and the semiclassical EBK quantization correspond to eigenvalues in the direct neighborhood of the focus line $F$ in Fig. 17. A more refined semiclassical approach carried out in [33] shows that even the quality of the semiclassical eigenvalues close to $F$ can be improved (see also [8]).

\subsection{Monodromy of the Quantum Spectrum}

In order to see the quantum monodromy we have to look at the quantum mechanical spectrum in the space of $\left(E, K, L_{x}\right)$. This three-dimensional picture is however too complicated to be useful. Instead we choose to select certain subsets of states with eigenvalues located on two-dimensional planes and present them in different projections.

In the discussion of the $S L(3, \mathbb{Z})$ normal form of the monodromy matrix $\boldsymbol{M}$ in Section 3 we found that besides $I_{\varphi}$ the linear combination $I_{\eta}+2 I_{\xi}$ is invariant under $\boldsymbol{M}$. Note that the corresponding Maslov index has $\alpha_{\eta}+2 \alpha_{\xi}=0$ mod 4 so that both invariant actions have effective Maslov index 0 . We believe that this is a general fact. In particular, the vector of Maslov indices $\boldsymbol{\alpha}$ is an eigenvector 


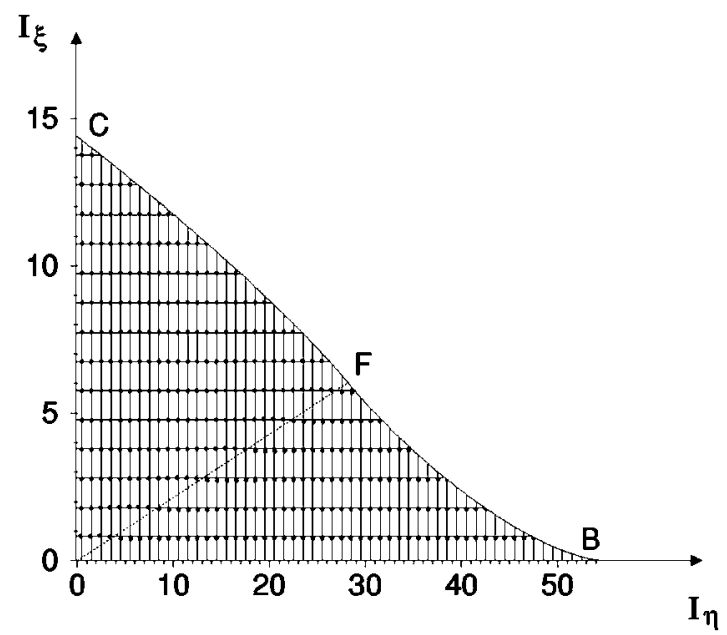

FIG. 17. Exact quantum mechanical eigenvalues and the lattice $\mathcal{L}_{\mathrm{EBK}}$ below the energy surface $E=2000$. $I_{\eta}$ and $I_{\xi}$ are measured in units of $\hbar ; I_{\varphi}=0$.

of all matrices with eigenvalue one,

$$
M_{1} \alpha=M_{2} \alpha=M \alpha=\alpha .
$$

A smooth two-dimensional plane in the space of the constants of motion is, e.g., obtained by inverting $I_{\eta}+2 I_{\xi}=n$ for $l_{\varphi} \geq 0$ with some constant $n \in \mathbb{N}$ and $I_{\eta}+2 I_{\xi}-2 I_{\varphi}=n$ with the same $n$ for $l_{\varphi} \leq 0$. The latter action is the second component of the image of the natural actions $\left(I_{\varphi}, I_{\eta}, I_{\xi}\right)$ under both $\boldsymbol{M}_{1}$ and $\boldsymbol{M}_{2}$. Note that the inverse of both matrices $\boldsymbol{M}_{1}$ and $\boldsymbol{M}_{2}$ map this action back to $I_{\eta}+2 I_{\xi}$ in accordance with our statement that this action is invariant under the monodromy matrix $\boldsymbol{M}$ which relates smooth actions for a full cycle about the line $F$. In Fig. 19a we represent

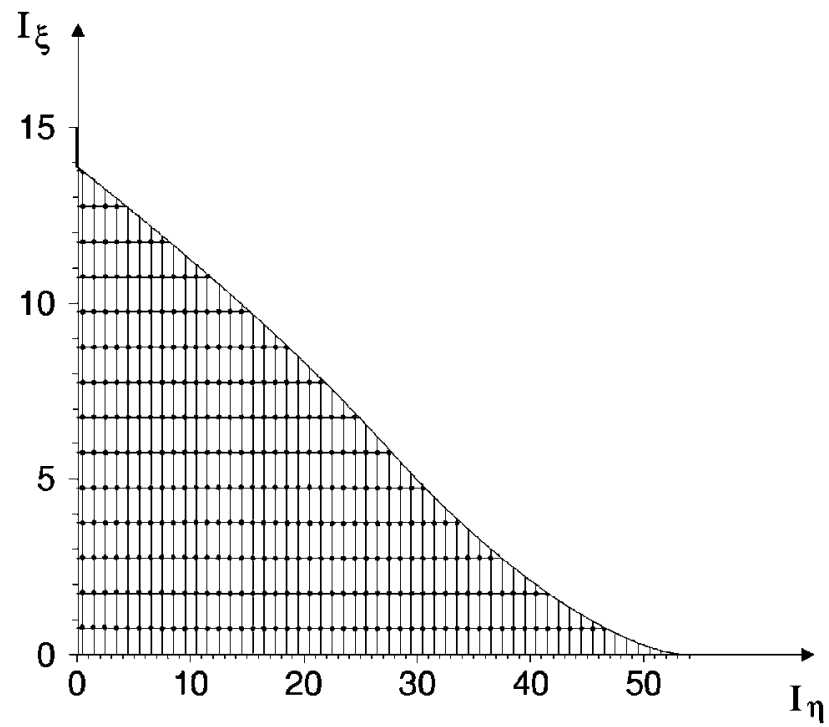

FIG. 18. Analogue of Fig. 17 for $I_{\varphi}=\hbar$. 
a)

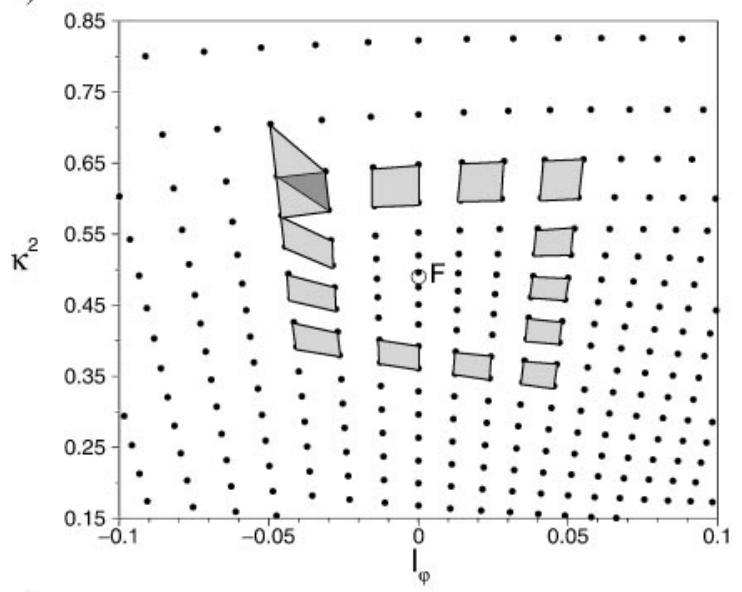

b)

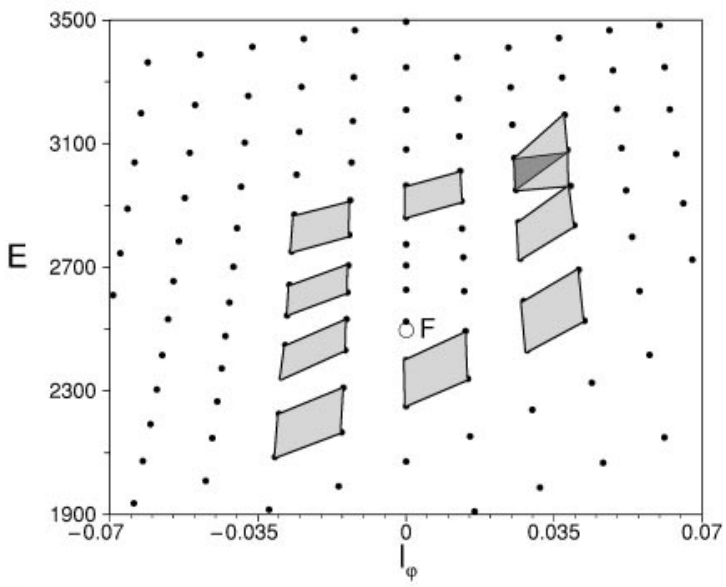

c)

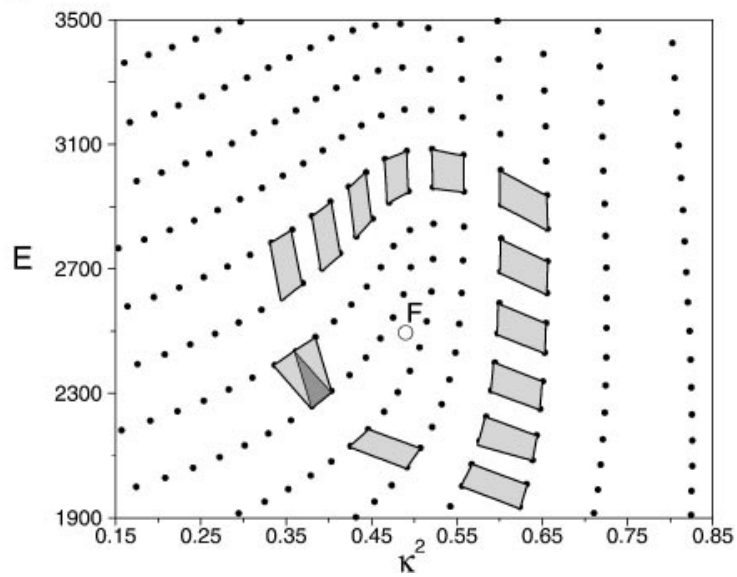

FIG. 19. Projections of the quantum mechanical spectrum in the plane $I_{\eta}+2 I_{\xi}=45$ for $l_{\varphi} \geq 0$ and $I_{\eta}+2 I_{\xi}-2 I_{\varphi}=45$ for $l_{\varphi} \leq 0$ to the planes $\left(l_{\varphi}, \kappa^{2}\right)(\mathrm{a}),\left(l_{\varphi}, E\right)(\mathrm{b})$, and $\left(\kappa^{2}, E\right)(\mathrm{c})$. The circle $F$ marks the intersection with the isolated line of the bifurcation diagram. 
the eigenvalues of the quantum states obtained this way for $n=45$ in the plane of the energy scaled constants of motion $l_{\varphi}$ and $\kappa^{2}$. The subset contains an infinite number of states of which Fig. 19a shows only a small portion with eigenvalues close to $F$. Locally the existence of a lattice from the $E B K$ quantization is well pronounced. The lattice lines in vertical direction correspond to the $E B K$ quantum number $n_{\varphi}$. Equivalently to the ambiguity of the "bad" action, there exists an infinite number of choices of locally smooth lattice lines or $E B K$ quantum numbers transversally to the lines $n_{\varphi}=$ const. The quantum monodromy becomes apparent if a lattice cell is parallel-transported along a cycle about $F$. The parallel-transport is defined through shifts along a local $E B K$ lattice. How a lattice cell is mapped after a full cycle is described by the monodromy matrix $\boldsymbol{M}$. Due to its non-trivial entry 1 there appears a shearing of the image lattice cell against the preimage lattice cell by one lattice site. The effect is similarly observed if the same plane is projected to the other two combinations of the constants of motion; see Figs. 19b and 19c. Due to the orientation of the plane of the eigenvalues in the three-dimensional space of the constants of motion the sense of rotation of a cycle about $F$ in Fig. 19b is opposite to the sense of rotation in Figs. 19a and 19c.

All states are a union of the slices in Fig. 19. Within each slice we observe monodromy with index 1 . For slices of states as, e.g., $I_{\eta}=$ const or $I_{\xi}=$ const the monodromy becomes an essentially three-dimensional effect, because the slice is not invariant. This is discussed and illustrated in detail in [34].

The eigenvalue lattice suggests that for states close to the circular billiard it is natural to replace the set of quantum numbers $\left(n_{\varphi}, n_{\eta}, n_{\xi}\right)$ for negative $n_{\varphi}$ by the new choice $\left(n_{\varphi}, n_{\eta}, n_{\xi}-n_{\varphi}\right)$ which gives a smooth lattice below $F$ in Fig. 19a. In contrast to that, the natural set of quantum numbers close to the geodesic flow, i.e., above $F$ in Fig. 19a, is obtained by $\left(n_{\varphi}, n_{\eta}-2 n_{\varphi}, n_{\xi}\right)$ for $n_{\varphi} \leq 0$. For a similar effect and its implications on physics see [35].

\section{CONCLUSION}

In the present paper we discussed the phenomenon of monodromy both classically and quantum mechanically for the billiard with a prolate ellipsoidal boundary. We showed that the monodromy here is similar to the monodromy extensively discussed for the case of a two degrees of freedom system with a focus point. The similarity becomes especially apparent in the discussion of the two-dimensional billiard map. The bifurcation diagram of the billiard map has an isolated point whose preimage in phase space is a singly pinched 2-torus. The existence of a pinched 2-torus is considered as the main aspect for the appearance of monodromy. The commonly used method of singular reduction [5] for calculating the number of pinches does not apply in the present case since the billiard is a non-smooth system whose phase space has a boundary. Instead we derived the monodromy directly from the consideration of actions of the full three degrees of freedom billiard flow for which the isolated point of the bifurcation diagram generalizes to an isolated line. Topologically, the preimage in phase space of each point on this line is the product of a pinched 2-torus and a circle.

Other than for the two degrees of freedom system the monodromy matrix calculated from the natural actions associated with the separating coordinates does not appear in a normal form where it has only one non-trivial off-diagonal element which immediately would give the index of the monodromy. Instead, the index is obtained from conjugation of the natural monodromy matrix by unimodular transformations. In particular, this yields a second action besides the angular momentum which is invariant under the monodromy. Constant values of this action define smooth two-dimensional surfaces in the three-dimensional space of the constants of motion. A two-dimensional picture of the quantum monodromy is most easily obtained by projecting such a surface to a plane spanned by either two of the constants of motion. In each projection the expected shearing of an EBK quantum lattice cell moved along a full cycle about the focus line is observed. 
The monodromy examined here is similar to the monodromy found in the two centers problem which separates in the same coordinate system [36]. In principle it is possible to analyze the monodromy for the prolate ellipsoidal billiard alone in terms of the two-dimensional billiard map. However, the full three degrees of freedom billiard is considered as a system of greater relevance to physics. For the two centers problem the necessity to deal with a three degrees of freedom system is even more apparent because there are two isolated lines to both of which there corresponds a monodromy matrix of index 1 . The actions that are invariant under the monodromy are different and therefore from a global point of view the monodromy is to be discussed as a truly three-dimensional effect.

The classical monodromy is connected to the collisions of branch points with a pole of the action integrals. Typically, the natural actions are not smooth under such a collision as we demonstrated in this paper for the natural actions related to the separating coordinate system in the limit of a vanishing angular momentum. In particular, the isolated point in the bifurcation diagram appears through a multiple collision of branch points with a pole. This suggests a general discussion of the analytic behavior of the action integrals in such a case. Such an analysis can be performed in terms of so-called Picard-Fuchs equations which are a linear set of equations giving the action integrals as functions of the constants of motion $[14,37]$. Such an analysis is currently under investigation. Quantum mechanically, the collision of branch points with poles becomes apparent in the separated Schrödinger equation. This observation may be crucial for developing a general monodromy theory for coupled Sturm-Liouville boundary value problems.

Ellipsoidal billiards can be viewed as singular versions of the well known integrable geodesic flow on ellipsoidal surfaces $[29,30]$ where the shortest semi-axis collapses to zero. It is to be expected that the monodromy is also apparent in these smooth systems and also in the related Neumann system $[38,39]$.

\section{ACKNOWLEDGMENTS}

H.R.D. was partially supported by the European Research and Training Network Mechanics and Symmetry MASIE.

\section{REFERENCES}

1. R. Cushman and J. J. Duistermaat, Bull. Amer. Math. Soc. 19 (1988), 475.

2. J. J. Duistermaat, Comm. Pure Appl. Math. 33 (1980), 687.

3. V. I. Arnold, "Mathematical Methods of Classical Mechanics," Graduate Texts in Mathematics, Vol. 60, Springer-Verlag, Berlin, 1978.

4. A. T. Fomenko, in "The Geometry of Hamiltonian Systems" (T. Ratiu, Ed.), p. 131, Springer-Verlag, New York, 1991.

5. R. H. Cushman and L. M. Bates, "Global Aspects of Classical Integrable Systems," Birkhäuser, Basel, 1997.

6. R. H. Cushman and D. A. Sadovskii, Phys. D 142(1-2) (2000), 166.

7. N. T. Zung, Differential Geom. Appl. 7(2) (1997), 123.

8. M. S. Child, J. Phys. A 31 (1998), 657.

9. V. N. San, Comm. Pure Appl Math. 53(2) (2000), 143.

10. H. Waalkens, J. Wiersig, and H. R. Dullin, Ann. Phys. (N.Y.) 260(1) (1997), 50.

11. H. Waalkens, J. Wiersig, and H. R. Dullin, Ann. Phys. (N.Y.) 276(1) (1999), 64.

12. P. H. Richter, "Die Theorie des Kreisels in Bildern," Report 226, Institut für Dynamische Systeme, 1990.

13. H. R. Dullin, M. Juhnke, and P. H. Richter, Bifurcation and Chaos 4(6) (1994), 1535.

14. H. R. Dullin, P. H. Richter, and A. P. Veselov, Regular Chaotic Dynam. 33 (1998), 18.

15. M. Brack, Rev. Mod. Phys. 65 (1993), 677.

16. M. Brack and R. K. Bhaduri, "Semiclassical Physics," Addison-Wesley, Reading, MA, 1997.

17. V. M. Strutinsky, A. G. Magner, S. R. Ofengenden, and T. Døssing, Z. Phys. A 283 (1977), 269.

18. A. G. Magner et al., Ann. Phys. 6 (1997), 555.

19. M. Fliesser et al., Phys. Rev. A 56 (1997), 4879.

20. P. M. Morse and H. Feshbach, "Methods of Theoretical Physics," McGraw-Hill, New York, 1953. 
21. A. P. Veselov, Uspekhi Mat. Nauk 46(5(281)) (1991), 3.

22. P. H. Richter, A. Wittek, M. P. Kharlamov, and A. P. Kharlamov, Z. Naturforsch. A 50 (1995), 693.

23. P. F. Byrd and M. D. Friedman, "Handbook of Elliptic Integrals for Engineers and Physicists," Springer-Verlag, Berlin, 1971.

24. G. H. Hardy and E. M. Wright, "An Introduction to the Theory of Numbers," 5th ed., Clarendon Oxford Univ. Press, New York, 1979.

25. M. Sieber, Nonlinearity 11 (1998), 1607.

26. H. R. Dullin, O. Heudecker, M. Juhnke, H. Pleteit, H.-P. Schwebler, H. Waalkens, J. Wiersig, and A. Wittek, "Energy Surfaces in Action Space,” Report 406, Institut für Dynamische Systeme, 1997.

27. J. B. Keller and S. I. Rubinow, Ann. Phys. (N.Y.) 9 (1960), 24.

28. Y. F. Lazutkin, Izv. Acad. Sci. Ser. Math. 37 (1973), 186.

29. C. G. J. Jacobi, “Vorlesungen über Dynamik,” Chelsea, New York, 1969.

30. J. Moser, Progr. Math. 8 (1980), 233.

31. H. A. Erikson and E. L. Hill, Phys. Rev. 75(1) (1949), 29.

32. M. Abramowitz and I. A. Stegun, "Handbook of Mathematical Functions," Dover, New York, 1965.

33. Y. Ayant and R. Arvieu, J. Phys. A. 20 (1987), 1115.

34. H. Waalkens, "Elliptic and Ellipsoidal Quantum Billiards," Shaker Verlag, Aachen, 1999.

35. M. S. Child, T. Weston, and J. Tennyson, Mol. Phys. 96 (1999), 371.

36. H. R. Dullin, A. Junge, and H. Waalkens, in preparation, 2001.

37. H. R. Dullin, P. H. Richter, A. Veselov, and H. Waalkens, Phys. D 155 (2001), 159.

38. C. Neumann, J. Reine Angew. Math. 3 (1859), 46.

39. A. P. Veselov, Math. Z. 216 (1994), 337. 